\title{
ADAPTIVE TESTS FOR PERIODIC SIGNAL DETECTION WITH APPLICATIONS TO LASER VIBROMETRY*
}

\author{
Magalie Fromont ${ }^{1}$ And CÉline LÉvy-LeduC ${ }^{2}$
}

\begin{abstract}
Initially motivated by a practical issue in target detection via laser vibrometry, we are interested in the problem of periodic signal detection in a Gaussian fixed design regression framework. Assuming that the signal belongs to some periodic Sobolev ball and that the variance of the noise is known, we first consider the problem from a minimax point of view: we evaluate the so-called minimax separation rate which corresponds to the minimal $l_{2}$-distance between the signal and zero so that the detection is possible with prescribed probabilities of error. Then, we propose a testing procedure which is available when the variance of the noise is unknown and which does not use any prior information about the smoothness degree or the period of the signal. We prove that it is adaptive in the sense that it achieves, up to a possible logarithmic factor, the minimax separation rate over various periodic Sobolev balls simultaneously. The originality of our approach as compared to related works on the topic of signal detection is that our testing procedure is sensitive to the periodicity assumption on the signal. A simulation study is performed in order to evaluate the effect of this prior assumption on the power of the test. We do observe the gains that we could expect from the theory. At last, we turn to the application to target detection by laser vibrometry that we had in view.
\end{abstract}

Mathematics Subject Classification. 62G10, 62G08, 62G20.

Received September 11, 2004.

\section{INTRODUCTION}

One of the most topical issues in optronics is the identification of a target through the determination of some of its vibration parameters such as its vibration period for instance. The use of coherent lasers has provided some progress in this field. After emission of a continuous coherent laser wave, reflection of it on a target composed of reflectors vibrating at the same frequency, reception and demodulation, one receives a signal which consists of a deterministic periodic function corrupted by Gaussian white noise. It turns out that the period of the deterministic part of the signal is precisely the period of the target vibrations. Estimating this parameter would hence allow to identify the target. We are interested here in a step which has to precede this estimation phase, namely the target detection step.

Keywords and phrases. Periodic signal detection, adaptive test, minimax separation rates, nonparametric regression.

* The authors wish to express their thanks to Yannick Baraud and Pascal Massart for many interesting and fruitful discussions.

They are also grateful to the company Thalès Optronique which has supported this study.

1 Université Rennes II, Place du Recteur H. Le Moal, CS 24307, 35043 Rennes cedex, France; magalie.fromont@uhb.fr

2 Université Paris-Sud, Bât. 425, 91405 Orsay; France; celine.levy-leduc@math.u-psud.fr 
Taking into account the form of the observed signal in this laser vibrometry context, we consider the following general periodic fixed design regression model:

$$
Y_{j}=f\left(\frac{j}{n}\right)+\sigma \varepsilon_{j} \text { for } j \in\{1, \ldots, n\},
$$

where $f$ is some unknown real valued periodic function called the signal, the $\varepsilon_{j}$ 's are independent standard Gaussian random variables and $\sigma$ is a positive real number. We aim at testing the null hypothesis $\left(H_{0}\right)$ : " $f=0$ " against the alternative $\left(H_{1}\right):$ : $f \neq 0$ ", which amounts in the practical situation of our interest to detecting the presence of any target.

We particularly want to make the best possible use of the periodicity properties of the signal, keeping in mind of course that at this stage, we do not have any knowledge of the period itself. To do this, we have to know accurately the impact of such properties in our signal detection problem.

From a theoretical point of view, when $\sigma$ is known, the performances of a level $\alpha$ test of $\left(H_{0}\right)$ can be evaluated in terms of uniform separation rates with respect to some prescribed norm $\|$.$\| over classes of smooth functions.$ Given $\delta \in] 0,1$, a class of functions $\mathcal{S}$ and a level $\alpha$ test $\Phi_{\alpha}$ with values in $\{0,1\}$ rejecting $\left(H_{0}\right)$ when $\Phi_{\alpha}=1$, the uniform separation rate $\rho\left(\Phi_{\alpha}, \mathcal{S}, \delta, \sigma\right)$ of $\Phi_{\alpha}$ over the class $\mathcal{S}$ is defined as the smallest number $\rho$ such that the test guarantees a power at least equal to $(1-\delta)$ for all alternatives $f$ in $\mathcal{S}$ at a distance $\rho$ from 0 . More precisely, if $\mathbb{P}_{f}$ denotes the distribution of the observation $Y=\left(Y_{1}, \ldots, Y_{n}\right)$ defined by (1),

$$
\begin{aligned}
\rho\left(\Phi_{\alpha}, \mathcal{S}, \delta, \sigma\right) & =\inf \left\{\rho>0, \inf _{f \in \mathcal{S},\|f\| \geq \rho} \mathbb{P}_{f}\left[\Phi_{\alpha}=1\right] \geq 1-\delta\right\} \\
& =\inf \left\{\rho>0, \sup _{f \in \mathcal{S},\|f\| \geq \rho} \mathbb{P}_{f}\left[\Phi_{\alpha}=0\right]<\delta\right\} .
\end{aligned}
$$

We equip [0,1] with the measure $\mu_{n}$ given by $\mu_{n}=n^{-1} \sum_{j=1}^{n} \Delta_{j / n}, \Delta$. being the Dirac measure.

The first purpose of this paper is to evaluate the quantity

$$
\tilde{\rho}(\mathcal{S}, \alpha, \delta, \sigma)=\inf _{\Phi_{\alpha}} \rho\left(\Phi_{\alpha}, \mathcal{S}, \delta, \sigma\right)
$$

where the infimum is taken over all level $\alpha$ tests $\Phi_{\alpha}$ and $\rho$ is the uniform separation rate with respect to the usual norm of $\mathbb{L}_{2}\left([0,1], \mu_{n}\right), \mathcal{S}$ being some periodic Sobolev ball with a known smoothness parameter. This quantity introduced by Baraud in [1] as the $(\alpha, \delta)$-minimax rate of testing over $\mathcal{S}$ or the minimax separation rate over $\mathcal{S}$ is the non-asymptotic version of the (asymptotic) minimax rate of testing usually considered. The key reference for the computation of minimax rates of testing in various statistical models is the series of papers due to Ingster [11,12]. In the Gaussian white noise model, which is originally considered to study the problem of signal detection, Ingster establishes the minimax rates of testing with respect to the $\mathbb{L}_{r}-$ norm $(1 \leq r \leq+\infty)$ over some classes of alternatives like Sobolev, Hölder balls in $\mathbb{L}_{p}(p \geq r$ if $r \leq 2, p=r$ if $r>2)$ or ellipsoids in $l_{r}$. In particular, for Sobolev or Hölder balls with smoothness degree $s$, he obtains a rate with respect to the $\mathbb{L}_{2}-$ norm of order $n^{-2 s /(4 s+1)}$. Other kinds of alternatives such as Besov balls $B_{s, p, q}(R)$ with $\left.p \in\right] 0,2[$ for the $\mathbb{L}_{2}$-norm are considered by Lepski and Spokoiny in [15]. Furthermore, Lepski and Tsybakov in [16] extend Ingster's study by finding the exact separation constants over Hölder and Sobolev classes for the $\mathbb{L}_{\infty}-$ norm. Baraud in [1] focuses on the Gaussian sequence model and gives the minimax separation rates with respect to the $l_{2}$-norm over some ellipsoids in $l_{p}$ (with $\left.\left.p \in\right] 0,2\right]$ ) and some Besov bodies. His results, which allow (as explained by Spokoiny in [19]) to recover some of the results obtained in the Gaussian white noise model, can be distinguished by their non-asymptotic characteristic. As for the fixed design regression model, Härdle and Kneip in [9] state a first minimax result over some particular Sobolev classes. Gayraud and Pouet in [7] consider the more general problem of testing composite null hypotheses such as " $f$ belongs to some parametric family". They give the minimax rates of testing for this problem with respect to the $\mathbb{L}_{2}$-norm over some Hölder classes 
and prove that these rates are of the same order as those obtained for simple hypotheses in the Gaussian white noise model (see [12]).

The particularity of our work on this topic lies in the fact that we exhibit how the minimax separation rates in a periodic fixed design regression model depend on the period of the signal.

The second purpose of this paper is to propose a testing procedure which does not use any prior information on the smoothness of the signal $f$ and which is available when the variance of the noise and the period of $f$ are unknown. The problem of finding adaptive (assumption free) tests of " $f=0$ " is studied from a minimax point of view by Spokoiny [19,20] in the Gaussian white noise model over some Besov balls and by Baraud [1] in the Gaussian sequence model when the variance is known over some ellipsoids. They prove that adaptation is impossible without some loss of efficiency within an extra $\log \log n$ factor and they propose adaptive testing procedures which are rate optimal over several classes of alternatives simultaneously. In the fixed design regression model without any periodicity assumption, various testing procedures using no prior assumption on the smoothness of the signal $f$ have been proposed. Horowitz and Spokoiny in [10] use kernel smoothing methods to construct a procedure for the problem of testing that the signal belongs to some parametric family of functions. This test achieves a rate of order $(\sqrt{\log \log n} / n)^{2 s /(4 s+1)}$ for the normalized $l_{2}$-norm over Hölder, Sobolev and Besov classes of alternatives with smoothness parameter $s$. Besides, some methods of model selection by minimization of a penalized criterion allow to construct other assumption free testing procedures. The test proposed by Eubank and Hart in [5] is built from a criterion connected with Mallows' $C_{p}$. It achieves the parametric rate of testing over directional alternatives. The test developed by Baraud, Huet and Laurent in [2] is based on a criterion related to the one used by Laurent and Massart in [13] to estimate quadratic functionals in the regression framework. It consists in a multiple testing procedure which can be described as follows. Introduce some suitable collection of linear subspaces $\left\{S_{m}, m \in \mathcal{M}\right\}$ of $\mathbb{R}^{n}$ based on orthonormal functions of $\mathbb{L}_{2}([0,1])$ such that for each $m, S_{m} \neq\{0\}$ and $S_{m} \neq \mathbb{R}^{n}$, and some collection $\left\{\alpha_{m}, m \in \mathcal{M}\right\}$ of numbers in $] 0,1[$. Consider, for each $m$ in $\mathcal{M}$, the Fisher test with level $\alpha_{m}$ of the null hypothesis " $(f(1 / n), \ldots, f(1))^{T}=0$ " against the alternative " $(f(1 / n), \ldots, f(1))^{T} \in S_{m}$ ". One thus obtains a collection of Fisher tests and one decides to reject the null hypothesis if one of the tests of the collection does. From a theoretical point of view, Baraud, Huet and Laurent evaluate in [2] the quality of their test by the means of uniform separation rates over some Hölder balls with respect to the normalized $l_{2}-$ norm. They obtain some results similar to Horowitz and Spokoiny's ones, except that they look at the problem from a non-asymptotic angle. From a practical point of view, they illustrate their results by a simulation study. In the fixed design regression model with a periodic regression function, we can at least distinguish two kinds of signal detection procedures: some rely on a likelihood ratio test and others use Bayesian techniques. The ones based on likelihood ratio allow to test the null hypothesis $\left(H_{0}\right)$ against $\left(H_{1}\right)$ when the periodic regression function is a trigonometric polynomial with a not necessarily known degree. Such procedures are developed in the books of Brockwell and Davis [4] p. 337 et seq. or in the one of Quinn and Thomson [18] p. 73 et seq. They consist in rejecting the null hypothesis if the periodogram of the observations contains a value substantially larger than the average value, recalling that the periodogram of $Y=\left(Y_{1}, \ldots, Y_{n}\right)$ is defined by $I_{Y}(q)=n^{-1}\left|\sum_{j=1}^{n} \mathrm{e}^{-2 i \pi j q / n} Y_{j}\right|^{2}$ for $q \in\{1, \ldots, n-1\}$. As for the Bayesian methods, they are detailed by Gregory and Loredo in [8]. The relevance of these different tests is essentially justified by simulation studies.

Our main concern is to provide a testing procedure which adapts both to the smoothness and the period of the signal. Our approach consists in fact in using the above procedure due to Baraud, Huet and Laurent [2] with a precise collection of models $\left\{S_{m}, m \in \mathcal{M}\right\}$ which takes into account different possible values of the periodicity parameter. We evaluate the uniform separation rates of the obtained test over some periodic Sobolev balls with respect to the usual norm of $\mathbb{L}_{2}\left([0,1], \mu_{n}\right)$. In particular, we point out in Section 2 that they are of the same order, up to a logarithmic factor, as the minimax separation rates that we establish in Section 1. An experimental comparison between the powers of the considered test and the one originally investigated by Baraud, Huet and Laurent in [2] (which does not take any periodicity assumption into account) is presented in Section 3. In Section 4, we finally focus on the application to target detection by laser vibrometry which has 
initially motivated this work. The proofs of the theorems and the propositions stated in the paper are detailed in Section 6.

\section{Minimax Separation Rates over Periodic Sobolev Balls}

Throughout this section, we assume that $\sigma$ is known. Given $k \in\{1, \ldots, n\}, s \in \mathbb{N}^{*}$ and $R>0$, we consider the periodic Sobolev ball:

$$
\mathcal{S}_{k}(s, R)=\left\{f \in \mathcal{C}^{s}([0,1]), f \text { is periodic with period } k / n,\left\|f^{(s)}\right\|_{2, k / n} \leq R\right\},
$$

where $\mathcal{C}^{s}([0,1])$ denotes the set of functions $f$ from $[0,1]$ with a continuous $s$-th order derivative denoted by $f^{(s)}$, and for $\left.\theta \in\right] 0,1\left[\right.$, for all $g$ in $\mathbb{L}_{2}([0,1], \mathrm{d} x)$,

$$
\|g\|_{2, \theta}^{2}=\theta^{-1} \int_{0}^{\theta} g^{2}(x) \mathrm{d} x
$$

Let us introduce the usual norm $\|\cdot\|_{n}$ of $\mathbb{L}_{2}\left([0,1], \mu_{n}\right)$ given, for all $g$ in $\mathbb{L}_{2}\left([0,1], \mu_{n}\right)$, by

$$
\|g\|_{n}^{2}=\frac{1}{n} \sum_{j=1}^{n} g^{2}\left(\frac{j}{n}\right)
$$

Fixing some levels $\alpha \in] 0,1[$ and $\delta \in] 0,1-\alpha[$, our aim in this section is to evaluate the minimax separation rate over $\mathcal{S}_{k}(s, R)$ defined by

$$
\tilde{\rho}\left(\mathcal{S}_{k}(s, R), \alpha, \delta, \sigma\right)=\inf _{\Phi_{\alpha}} \inf \left\{\rho>0, \sup _{f \in \mathcal{S}_{k}(s, R),\|f\|_{n} \geq \rho} \mathbb{P}_{f}\left[\Phi_{\alpha}=0\right]<\delta\right\}
$$

where the infimum is taken over all level $\alpha$ tests $\Phi_{\alpha}$ rejecting " $f=0$ " when $\Phi_{\alpha}=1$.

\subsection{Lower bound}

Following a general idea due to Ingster, we derive in this section a lower bound for $\tilde{\rho}\left(\mathcal{S}_{k}(s, R), \alpha, \delta, \sigma\right)$.

For all $\mathcal{B} \subset \mathbb{L}_{2}\left([0,1], \mu_{n}\right)$, we define

$$
\beta(\mathcal{B})=\inf _{\Phi_{\alpha}} \sup _{f \in \mathcal{B}} \mathbb{P}_{f}\left(\Phi_{\alpha}=0\right)
$$

In the following, for every $x, y$ in $\mathbb{R}$, we set $x \wedge y=\inf \{x, y\} . c$ denotes a constant that may vary from line to line. The dependency of $c$ with respect to various parameters is specified by the notation $c($.$) .$

Theorem 1. Given some levels $\alpha \in] 0,1[$ and $\delta \in] 0,1-\alpha[$, there exist some absolute constant $\kappa \in] 0,1]$ and some positive constant $c(s)$ such that for every integer $k$ in $\left[\kappa^{-1}, n / 2\right]$, setting

$$
\rho_{k}^{2}(s, R)=c(s) \sup _{D \in\{1, \ldots,[\kappa k]\}}\left(\frac{(1-\alpha-\delta) \sqrt{D} \sigma^{2}}{n} \wedge R^{2} D^{-2 s}\left(\frac{k}{n}\right)^{2 s}\right)
$$

one has, with $\beta$ defined by (4),

$$
\beta\left(\left\{f \in \mathcal{S}_{k}(s, R),\|f\|_{n}^{2} \geq \rho_{k}^{2}(s, R)\right\}\right) \geq \delta .
$$

This theorem allows us to obtain the following explicit lower bound for $\tilde{\rho}\left(\mathcal{S}_{k}(s, R), \alpha, \delta, \sigma\right)$. 
Corollary 1. Given some levels $\alpha \in] 0,1[$ and $\delta \in] 0,1-\alpha[$, there exist some absolute constant $\kappa \in] 0,1]$ and some positive constant $c(s, \alpha, \delta)$ such that for every integer $k$ in $\left[\kappa^{-1}, n / 2\right]$,

$$
\tilde{\rho}^{2}\left(\mathcal{S}_{k}(s, R), \alpha, \delta, \sigma\right) \geq c(s, \alpha, \delta)\left(R^{\frac{2}{1+4 s}}\left(\frac{k}{n}\right)^{\frac{2 s}{4 s+1}}\left(\frac{\sigma^{2}}{n}\right)^{\frac{4 s}{4 s+1}} \wedge \frac{\sqrt{k} \sigma^{2}}{n} \wedge R^{2}\left(\frac{k}{n}\right)^{2 s}\right) .
$$

In particular, for all $k \in\left[\kappa^{-1}, n / 2\right]$, if

$$
\frac{\sigma}{\sqrt{n}}\left(\frac{k}{n}\right)^{-s} \leq R \leq \sigma n^{s-1 / 4}\left(\frac{k}{n}\right)^{1 / 4}
$$

then

$$
\tilde{\rho}\left(\mathcal{S}_{k}(s, R), \alpha, \delta, \sigma\right) \geq c^{\prime}(s, \alpha, \delta) R^{\frac{1}{1+4 s}}\left(\frac{k}{n}\right)^{\frac{s}{4 s+1}}\left(\frac{\sigma^{2}}{n}\right)^{\frac{2 s}{4 s+1}} .
$$

Proof: It is easy to see that the result

$$
\beta\left(\left\{f \in \mathcal{S}_{k}(s, R),\|f\|_{n}^{2} \geq \rho_{k}^{2}(s, R)\right\}\right) \geq \delta
$$

of Theorem 1 leads to the inequality:

$$
\tilde{\rho}^{2}\left(\mathcal{S}_{k}(s, R), \alpha, \delta, \sigma\right) \geq \rho_{k}^{2}(s, R)
$$

Then, we only need to compute an explicit lower bound for $\rho_{k}^{2}(s, R)$.

Let us prove that for $\kappa^{-1} \leq k \leq n / 2$,

$$
\sup _{D \in\{1, \ldots,[\kappa k]\}}\left(R^{2}\left(\frac{k}{n}\right)^{2 s} D^{-2 s} \wedge \frac{\sqrt{D}}{n} \sigma^{2}\right) \geq \frac{1}{\sqrt{2}} R^{\frac{2}{1+4 s}}\left(\frac{k}{n}\right)^{\frac{2 s}{1+4 s}}\left(\frac{\sigma^{2}}{n}\right)^{\frac{4 s}{4 s+1}} \wedge \frac{\sqrt{[\kappa k]} \sigma^{2}}{n} \wedge R^{2}\left(\frac{k}{n}\right)^{2 s} .
$$

Remark that $R^{2}(k / n)^{2 s} D^{-2 s} \leq \sqrt{D} \sigma^{2} / n$ if and only if $D \geq D^{*}$, where $D^{*}$ is the smallest integer at least equal to $\left((k / n)^{2 s} n R^{2} / \sigma^{2}\right)^{2 /(1+4 s)}$, that is

$$
D^{*}=\inf \left\{D \in \mathbb{N}, D \geq\left(\left(\frac{k}{n}\right)^{2 s} \frac{n R^{2}}{\sigma^{2}}\right)^{\frac{2}{1+4 s}}\right\} .
$$

We can distinguish three cases.

If $D^{*}=1$, for all $D$ in $\mathcal{D}_{k}=\{1, \ldots,[\kappa k]\}, D \geq D^{*}$, which implies that $R^{2}(k / n)^{2 s} D^{-2 s} \leq \sqrt{D} \sigma^{2} / n$. Hence,

$$
\sup _{D \in \mathcal{D}_{k}}\left(R^{2}\left(\frac{k}{n}\right)^{2 s} D^{-2 s} \wedge \frac{\sqrt{D}}{n} \sigma^{2}\right)=R^{2}\left(\frac{k}{n}\right)^{2 s} \text {. }
$$

If $D^{*}>[\kappa k]$, for all $D$ in $\mathcal{D}_{k}, \sqrt{D} \sigma^{2} / n<R^{2}(k / n)^{2 s} D^{-2 s}$, and

$$
\sup _{D \in \mathcal{D}_{k}}\left(R^{2}\left(\frac{k}{n}\right)^{2 s} D^{-2 s} \wedge \frac{\sqrt{D}}{n} \sigma^{2}\right)=\frac{\sqrt{[\kappa k]} \sigma^{2}}{n} .
$$

Finally, let us see what happens when $2 \leq D^{*} \leq[\kappa k]$. Since in this case $\left(D^{*}-1\right) \in \mathcal{D}_{k}$, we have that

$$
\sup _{D \in \mathcal{D}_{k}}\left(R^{2}\left(\frac{k}{n}\right)^{2 s} D^{-2 s} \wedge \frac{\sqrt{D}}{n} \sigma^{2}\right) \geq R^{2}\left(\frac{k}{n}\right)^{2 s}\left(D^{*}-1\right)^{-2 s} \wedge \frac{\sqrt{D^{*}-1}}{n} \sigma^{2} .
$$


So, from the definition of $D^{*}$ and the fact that $D^{*} \geq 2$ leads to $D^{*}-1 \geq D^{*} / 2$, we derive:

$$
\sup _{D \in \mathcal{D}_{k}}\left(R^{2}\left(\frac{k}{n}\right)^{2 s} D^{-2 s} \wedge \frac{\sqrt{D}}{n} \sigma^{2}\right) \geq \frac{\sqrt{D^{*}}}{\sqrt{2} n} \sigma^{2} \geq \frac{1}{\sqrt{2}} R^{\frac{2}{1+4 s}}\left(\frac{k}{n}\right)^{\frac{2 s}{1+4 s}}\left(\frac{\sigma^{2}}{n}\right)^{\frac{4 s}{4 s+1}}
$$

which concludes the proof.

\subsection{Upper bound}

We now aim at establishing the optimality of the above lower bound, provided that the radius $R$ of the periodic Sobolev ball $\mathcal{S}_{k}(s, R)$ belongs to some specified interval. For all $\theta>0$, we introduce the Fourier basis on $[0, \theta]$ defined as follows:

$$
\left\{\begin{array}{cl}
\varphi_{\theta, 1}(x) & =1 \\
\varphi_{\theta, 2 p}(x) & =\sqrt{2} \cos (2 \pi p x / \theta), \forall p \geq 1 \\
\varphi_{\theta, 2 p+1}(x) & =\sqrt{2} \sin (2 \pi p x / \theta), \forall p \geq 1
\end{array}\right.
$$

For all $D$ in $\mathbb{N}^{*}$, we consider the linear subspace $S_{D}$ of $\mathbb{R}^{n}$ spanned by the vectors:

$$
\left\{\left(\varphi_{k / n, l}(1 / n), \ldots, \varphi_{k / n, l}(1)\right)^{T}, l=1, \ldots, D\right\} .
$$

Let us denote by $\Pi_{S_{D}}$ the orthogonal projection onto $S_{D}$ with respect to the Euclidean norm of $\mathbb{R}^{n}$ and introduce the norm $\|\cdot\|_{\mathbb{R}^{n}}$ given by:

$$
\|V\|_{\mathbb{R}^{n}}^{2}=\frac{1}{n} \sum_{j=1}^{n} V_{j}^{2} \text { for all } V=\left(V_{1}, \ldots, V_{n}\right)^{T} \in \mathbb{R}^{n} .
$$

Proposition 1. Let $\alpha \in] 0,1[$ and $\delta \in] 0,1-\alpha[$. Define

$$
D_{k}^{*}=k \wedge \inf \left\{D \in \mathbb{N}, D \geq\left((k / n)^{2 s} n R^{2} / \sigma^{2}\right)^{2 /(1+4 s)}\right\}
$$

and denote by $D_{k}$ the dimension of the linear space $S_{D_{k}^{*}}$. Let

$$
\Phi_{\alpha}^{*}=\mathbb{I}_{\left\{n\left\|\Pi_{S_{D_{k}^{*}}}(Y)\right\|_{\mathbb{R}^{n}}^{2}>t_{D_{k}, \alpha} \sigma^{2}\right\}},
$$

where $t_{D_{k}, \alpha}$ is the $(1-\alpha)$ quantile of the $\chi^{2}$ distribution with $D_{k}$ degrees of freedom. There exists some positive constant $c(\alpha, \delta)$ such that the test $\Phi_{\alpha}^{*}$ satisfies:

$$
\mathbb{P}_{0}\left(\Phi_{\alpha}^{*}=1\right) \leq \alpha, \quad \text { and } \quad \mathbb{P}_{f}\left(\Phi_{\alpha}^{*}=0\right) \leq \delta
$$

for all $f \in \mathcal{S}_{k}(s, R)$ such that:

$$
\|f\|_{n}^{2} \geq c(\alpha, \delta)\left(R^{\frac{2}{1+4 s}}\left(\frac{k}{n}\right)^{\frac{2 s}{4 s+1}}\left(\frac{\sigma^{2}}{n}\right)^{\frac{4 s}{4 s+1}}+\frac{R^{2} k}{n^{2 s}}+\frac{\sigma^{2}}{n}\right) .
$$

In particular, if

then

$$
\frac{\sigma}{\sqrt{n}}\left(\frac{k}{n}\right)^{-s} \leq R \leq \sigma n^{\frac{8 s^{2}-6 s-1}{8 s}}\left(\frac{k}{n}\right)^{-\frac{2 s+1}{8 s}}
$$

$$
\tilde{\rho}\left(\mathcal{S}_{k}(s, R), \alpha, \delta, \sigma\right) \leq c^{\prime}(\alpha, \delta) R^{\frac{1}{1+4 s}}\left(\frac{k}{n}\right)^{\frac{s}{4 s+1}}\left(\frac{\sigma^{2}}{n}\right)^{\frac{2 s}{4 s+1}}
$$


Comments.

(i) Assuming that $k$ belongs to $\left[\kappa^{-1}, n / 2\right]$ and that the radius $R$ belongs to the range given by (8), we derive from Corollary 1 and Proposition 1 that the minimax separation rate $\tilde{\rho}\left(\mathcal{S}_{k}(s, R), \alpha, \delta, \sigma\right)$ for the considered problem over $\mathcal{S}_{k}(s, R)$ is of order

$$
R^{\frac{1}{1+4 s}}\left(\frac{k}{n}\right)^{\frac{s}{4 s+1}}\left(\frac{\sigma^{2}}{n}\right)^{\frac{2 s}{4 s+1}} .
$$

Introducing the larger class $\mathcal{S}_{k}^{\prime}(s, R)=\left\{f, f\right.$ is periodic with period $k / n$ and $f(x)=\sum_{l>1} \beta_{l}(f) \varphi_{k / n, l}(x)$ with $\left.(2 \pi)^{2 s} \sum_{l>2}[l / 2]^{2 s} \beta_{l}^{2}(f) \leq R^{2}(k / n)^{2 s}\right\}$, one can easily see that the same result holds for $\tilde{\rho}\left(\mathcal{S}_{k}^{\prime}(s, R), \alpha, \delta, \sigma\right)$. Such a result is in fact comparable to the minimax separation rate that one can obtain in the usual Gaussian white noise model (see Appendix p. 73 for further details).

(ii) The range defined by (8) for the radius $R$ is not so restrictive when we consider the rates from an asymptotic point of view. In this case, $k / n$ tends to a fixed period $\tau$ as $n$ tends to $\infty$, and since $s$ is a positive integer, any positive $R$ is allowed.

\section{AdAPtive testing PROCEDURES With UNKNOWN VARIANCE}

We turn here to the problem of finding adaptive tests of $\left(H_{0}\right)$ " $f=0$ " against $\left(H_{1}\right)$ " $f \neq 0$ " in $\mathbb{L}_{2}\left([0,1], \mu_{n}\right)$ when the variance $\sigma^{2}$ of the $Y_{j}$ 's given by (1) is unknown. We consider the following test of " $(f(1 / n), \ldots, f(1))^{T}=$ 0 " against " $(f(1 / n), \ldots, f(1))^{T} \neq 0$ " proposed by Baraud, Huet and Laurent in [2]. Let us fix some $\alpha$ in ]0,1[. Given some finite collection of linear subspaces $\left\{S_{m}, m \in \mathcal{M}\right\}$ of $\mathbb{R}^{n}$, let

$$
T_{\alpha}=\sup _{m \in \mathcal{M}}\left\{\frac{\left(n-D_{m}\right)\left\|\Pi_{m}(Y)\right\|_{\mathbb{R}^{n}}^{2}}{D_{m}\left\|Y-\Pi_{m}(Y)\right\|_{\mathbb{R}^{n}}^{2}}-\bar{F}_{D_{m}, n-D_{m}}^{-1}\left(u_{\alpha}\right)\right\}
$$

where:

- $\|.\|_{\mathbb{R}^{n}}$ is the norm defined by $(7)$;

- $\bar{F}_{D_{m}, n-D_{m}}(u)$ is the probability for a Fisher with $D_{m}$ and $n-D_{m}$ degrees of freedom variable to be larger than $u$;

- $\Pi_{m}$ is the orthogonal projection onto $S_{m}$ with respect to the Euclidean norm;

- $D_{m}$ is the dimension of $S_{m}$.

The null hypothesis " $(f(1 / n), \ldots, f(1))^{T}=0$ " is rejected when the test statistic $T_{\alpha}$ is positive.

We propose to use this testing procedure by taking particular collections of linear subspaces $\left\{S_{m}, m \in \mathcal{M}\right\}$ which fit the periodicity assumption on the signal. More precisely, when the period of the signal is known, the chosen collection of subspaces is rather similar to the one studied in details by Baraud, Huet and Laurent. When the period is unknown, the idea is to take a collection of subspaces corresponding to different values of the periodicity parameter. Our aim in the following two sections is to evaluate the uniform separation rates of the consequent tests over some Sobolev balls. We then use a result due to Baraud, Huet and Laurent [2] that we recall in Section 6 ( $c f$. Th. 2). The proof of this result is based on some deviations inequalities for noncentral $\chi^{2}$ variables due to Birgé [3] combined with some exponential inequalities for Fisher variables.

\subsection{Testing procedure when the period of the signal is known}

Throughout this section, we assume that $n \geq 3$ and that $f$ is periodic with period $k / n$ for $k$ in $\{3, \ldots, n\}$. We recall that $\left\{\varphi_{k / n, l}, l \geq 1\right\}$ denotes the Fourier basis on $[0, k / n]$. We take

$$
\mathcal{M}=\left\{1,2,2^{2}, \ldots, 2^{\left[\log _{2}(k / 2)\right]}\right\}
$$

and for $D$ in $\mathcal{M}, S_{D}$ as the linear subspace of $\mathbb{R}^{n}$ spanned by the vectors:

$$
\left\{\left(\varphi_{k / n, l}(1 / n), \ldots, \varphi_{k / n, l}(1)\right)^{T}, l=1, \ldots, D\right\}
$$


The following proposition gives upper bounds for the uniform separation rates of the corresponding testing procedure over the periodic Sobolev balls $\mathcal{S}_{k}(s, R)$ defined in Section 1.

Proposition 2. Let $n \geq 3$ and $k \in\{3, \ldots, n\}$. Given some levels $\alpha$ and $\delta \in] 0,1\left[\right.$, let $T_{\alpha}$ be the test statistic defined by (9) with $\mathcal{M}$ and $\left\{S_{m}, m \in \mathcal{M}\right\}$ chosen as in (10) and (11). Assume that $n$ is large enough so that $\alpha \geq e^{-n / 20} \log n / \log 2$ and $\delta \geq 2 e^{-n / 42}$. For all $s$ in $\mathbb{N}^{*}$, there exist some positive constants $c(\alpha, \delta)$ and $c(s, \alpha, \delta)$ such that for all $R>0$, if $f$ belongs to $\mathcal{S}_{k}(s, R)$ and satisfies:

$$
\|f\|_{n}^{2} \geq c(\alpha, \delta)\left\{R^{\frac{2}{4 s+1}}\left(\frac{k}{n}\right)^{\frac{2 s}{4 s+1}}\left(\frac{\sqrt{\log \log k} \sigma^{2}}{n}\right)^{\frac{4 s}{4 s+1}}+\frac{\log \log k}{n} \sigma^{2}\right\}+c(s, \alpha, \delta) \frac{R^{2} k}{n^{2 s}}
$$

then

$$
\mathbb{P}_{f}\left(T_{\alpha}>0\right) \geq 1-\delta
$$

In particular, if

$$
\frac{\sigma}{\sqrt{n}}\left(\frac{k}{n}\right)^{-s}(\log \log k)^{s+1 / 2} \leq R \leq \sigma n^{\frac{8 s^{2}-6 s-1}{8 s}}\left(\frac{k}{n}\right)^{-\frac{2 s+1}{8 s}}(\log \log k)^{1 / 4}
$$

then

$$
\rho\left(\mathbb{I}_{\left\{T_{\alpha}>0\right\}}, \mathcal{S}_{k}(s, R), \delta, \sigma\right) \leq c^{\prime}(s, \alpha, \delta) R^{\frac{1}{1+4 s}}\left(\frac{k}{n}\right)^{\frac{s}{4 s+1}}\left(\frac{\sqrt{\log \log k} \sigma^{2}}{n}\right)^{\frac{2 s}{4 s+1}} .
$$

Comments.

According to the results of Section 1, this means that the testing procedure is rate optimal, up to a possible $\log \log k$ factor, over all the Sobolev balls $\mathcal{S}_{k}(s, R)$ such that (12) holds simultaneously. In view of the results due to Spokoiny [19] in the Gaussian white noise model for Besov balls and Baraud [1] in the Gaussian sequence model for families of nested ellipsoids, we can rightfully think that this loss of efficiency is unavoidable when we deal with an adaptive procedure. However, we have obtained no result which would confirm it.

\subsection{Testing procedure when the period of the signal is unknown}

We now consider the more realistic case where the period of the signal is unknown. Let us recall that $\left\{\varphi_{\theta, l}, l \geq 1\right\}$ denotes the Fourier basis on $[0, \theta]$. We consider the set

$$
\mathcal{M}=\left\{(k, D), k \in\{2, \ldots, n\}, D \in\left\{1,2, \ldots, 2^{\left[\log _{2}(k / 2)\right]}\right\}\right\}
$$

For $m=(k, D)$ in $\mathcal{M}$, we define $S_{m}$ as the linear space spanned by

$$
\left\{\left(\varphi_{k / n, l}(1 / n), \ldots, \varphi_{k / n, l}(1)\right)^{T}, l=1, \ldots, D\right\}
$$

In the following result, we establish upper bounds for the uniform separation rates of the corresponding procedure over some Sobolev balls described below.

Proposition 3. Let $n \geq 3$. Given some levels $\alpha$ and $\delta \in] 0,1\left[\right.$, let $T_{\alpha}$ be the test statistic defined by (9) with $\mathcal{M}$ and $\left\{S_{m}, m \in \mathcal{M}\right\}$ chosen as in (13) and (14). Introduce for $s \in \mathbb{N}^{*}, R>0$ and $\tau_{1} \in[2 / n, 1]$,

$$
\mathcal{S}\left(s, R, \tau_{1}\right)=\left\{f \in \mathcal{C}^{s}([0,1]), f \text { is periodic with period } \tau(f) \in\left[2 / n, \tau_{1}\right],\left\|f^{(s)}\right\|_{2, \tau(f)} \leq R\right\}
$$


Assume that $n$ is large enough so that $\alpha \geq \mathrm{e}^{-n / 20} \log (n !) / \log 2$ and $\delta \geq 2 \mathrm{e}^{-n / 42}$. For all $s$ in $\mathbb{N}^{*}$, there exists some positive constant $c(s, \alpha, \delta)$ such that for all $R>0, \tau_{1}$ in $[2 / n, 1]$, if $f$ belongs to $\mathcal{S}\left(s, R, \tau_{1}\right)$ and satisfies:

$$
\|f\|_{n}^{2} \geq c(s, \alpha, \delta)\left\{R^{\frac{2}{4 s+1}} \tau_{1}^{\frac{2 s}{4 s+1}}\left(\frac{\sqrt{\log (n)} \sigma^{2}}{n}\right)^{\frac{4 s}{4 s+1}}+\frac{\log (n)}{n} \sigma^{2}+\left(\frac{R^{2} \tau_{1}^{2 s}}{n \tau_{1}^{2}} \wedge R^{2} \tau_{1}^{2 s}\right) \log n\right\}
$$

then

$$
\mathbb{P}_{f}\left(T_{\alpha}>0\right) \geq 1-\delta
$$

In particular, if

$$
\begin{gathered}
\frac{\sigma}{\sqrt{n}} \tau_{1}^{-s}(\log n)^{s+1 / 2} \leq R \leq \frac{\sigma}{\sqrt{n}} \tau_{1}^{-s}\left(n \tau_{1}^{2}\right)^{\frac{4 s+1}{8 s}}(\log n)^{-\frac{2 s+1}{8 s}} \\
\rho\left(\mathbb{1}_{\left\{T_{\alpha>0}\right\}}, \mathcal{S}\left(s, R, \tau_{1}\right), \delta, \sigma\right) \leq c^{\prime}(s, \alpha, \delta) R^{\frac{1}{1+4 s}} \tau_{1}^{\frac{s}{4 s+1}}\left(\frac{\sqrt{\log n} \sigma^{2}}{n}\right)^{\frac{2 s}{4 s+1}} .
\end{gathered}
$$

Comments.

The upper bound for the uniform separation rate of the test over $\mathcal{S}\left(s, R, \tau_{1}\right)$ when $R$ and $\tau_{1}$ satisfy (15) is similar to the one obtained when the period of $f$ is known to be equal to $k / n$ (see Prop. 2), but with a loss of efficiency of order $\log n$ instead of $\log \log k$. This is technically due to the fact that we choose a collection $\left\{S_{m}, m \in \mathcal{M}\right\}$ which is rich enough to ensure that it contains, for any function $f$ with period $\tau(f)$ in $[2 / n, 1]$, some subspace $S_{m}$ close enough to $f$. Our choice for $\left\{S_{m}, m \in \mathcal{M}\right\}$ essentially relies on the properties of orthogonality of the family $\left\{\left(\varphi_{k / n, l}(1 / n), \ldots, \varphi_{k / n, l}(k / n)\right), 1 \leq l \leq k\right\}$ with respect to the Euclidean norm of $\mathbb{R}^{k}$. However, we do not know if the consequent extra $\log n$ factor is optimal or not.

\section{Simulation study}

We evaluate here the performances of the proposed test from a practical point of view. In particular, we want to highlight the improvement carried by the periodicity assumption. In the definition (9) of the test statistic $T_{\alpha}$, we have to choose the set $\mathcal{M}$ and the collection $\left\{S_{m}, m \in \mathcal{M}\right\}$ of linear subspaces onto which $Y$ is projected. For numerical reasons which are explained below, we do not only consider periods of the form $k / n$ : we propose to take

$$
\mathcal{M}=\left\{(q, D), q \in\{1, \ldots, Q\}, D \in\left\{2 d+1,0 \leq d \leq d_{0}\right\}\right\},
$$

where $Q$ and $d_{0}$ are some fixed integers with $2 d_{0} Q<n$, and for $m=(q, D)$ in $\mathcal{M}, S_{m}$ as the linear space spanned by the vectors

$$
\left\{\left(\varphi_{q^{-1}, l}(1 / n), \ldots, \varphi_{q^{-1}, l}(1)\right)^{T}, l=1, \ldots, D\right\} .
$$

With the same notations as in Section 2, we set

$$
T_{p e r}=\sup _{m \in \mathcal{M}}\left\{\frac{\left(n-D_{m}\right)\left\|\Pi_{m}(Y)\right\|_{\mathbb{R}^{n}}^{2}}{D_{m}\left\|Y-\Pi_{m}(Y)\right\|_{\mathbb{R}^{n}}^{2}}-\bar{F}_{D_{m}, n-D_{m}}^{-1}\left(u_{\alpha}\right)\right\},
$$

where

$$
u_{\alpha}=\sup \{u \in] 0,1\left[, \mathbb{P}_{0}\left(\sup _{m \in \mathcal{M}}\left\{\frac{\left(n-D_{m}\right)\left\|\Pi_{m}(Y)\right\|_{\mathbb{R}^{n}}^{2}}{D_{m}\left\|Y-\Pi_{m}(Y)\right\|_{\mathbb{R}^{n}}^{2}}-\bar{F}_{D_{m}, n-D_{m}}^{-1}(u)\right\}>0\right) \leq \alpha\right\} .
$$

Without taking the periodicity assumption into account, the corresponding statistic is

$$
T_{n p e r}=\sup _{m \in \mathcal{M}^{\prime}}\left\{\frac{\left(n-D_{m}\right)\left\|\Pi_{m}(Y)\right\|_{\mathbb{R}^{n}}^{2}}{D_{m}\left\|Y-\Pi_{m}(Y)\right\|_{\mathbb{R}^{n}}^{2}}-\bar{F}_{D_{m}, n-D_{m}}^{-1}\left(u_{\alpha}^{\prime}\right)\right\}
$$


where $\mathcal{M}^{\prime}=\left\{2 d+1,0 \leq d \leq d_{0}\right\}$, for $m=D$ in $\mathcal{M}^{\prime}, S_{m}$ is the linear space spanned by the vectors

$$
\left\{\left(\varphi_{1, l}(1 / n), \ldots, \varphi_{1, l}(1)\right)^{T}, l=1, \ldots, D\right\},
$$

and

$$
u_{\alpha}^{\prime}=\sup \{u \in] 0,1\left[, \mathbb{P}_{0}\left(\sup _{m \in \mathcal{M}^{\prime}}\left\{\frac{\left(n-D_{m}\right)\left\|\Pi_{m}(Y)\right\|_{\mathbb{R}^{n}}^{2}}{D_{m}\left\|Y-\Pi_{m}(Y)\right\|_{\mathbb{R}^{n}}^{2}}-\bar{F}_{D_{m}, n-D_{m}}^{-1}(u)\right\}>0\right) \leq \alpha\right\} .
$$

To implement the testing procedures based on $T_{p e r}$ and $T_{n p e r}$, the main point is to compute $\left\|\Pi_{(q, D)}(Y)\right\|_{\mathbb{R}^{n}}^{2}$ for every $(q, D)$ in $\mathcal{M}$. Considering

$$
A(q, D)=\left(A_{j, l}(q, D)\right)_{1 \leq j \leq n, 1 \leq l \leq D}=\left(\varphi_{q^{-1}, l}(j / n)\right)_{1 \leq j \leq n, 1 \leq l \leq D},
$$

we can see by straightforward calculations that when $q$ is an integer such that $2 d_{0} q<n, A^{T}(q, D) A(q, D)=n I_{D}$ $\left(I_{D}\right.$ denotes the identity matrix with dimension $\left.D\right)$. Minimizing the least squares criterion $\|Y-A(q, D) C\|_{\mathbb{R}^{n}}^{2}$ for $C$ in $\mathbb{R}^{n}$ then leads to $\Pi_{(q, D)}(Y)=n^{-1} A(q, D) A^{T}(q, D) Y$ and consequently,

$$
\left\|\Pi_{(q, D)}(Y)\right\|_{\mathbb{R}^{n}}^{2}=\frac{1}{n}\left\|A^{T}(q, D) Y\right\|_{\mathbb{R}^{n}}^{2}=\frac{1}{n^{2}} \sum_{l=1}^{D}\left(\sum_{j=1}^{n} \varphi_{q^{-1}, l}(j / n) Y_{j}\right)^{2} .
$$

Finally, if $D=2 d+1$, it is easy to see that:

$$
\left\|\Pi_{(q, D)}(Y)\right\|_{\mathbb{R}^{n}}^{2}=\frac{1}{n^{2}} \sum_{l=-d}^{d}\left|\sum_{j=1}^{n} \mathrm{e}^{-2 i \pi l j q / n} Y_{j}\right|^{2} .
$$

Such an expression is interesting for two reasons: first, it corresponds to a cumulated periodogram, the periodogram being a quantity frequently used in periodic signal detection, secondly, the values of $\sum_{j=1}^{n} \mathrm{e}^{-2 i \pi j m / n} Y_{j}$, for $m$ in $\{1, \ldots, n\}$, can be computed by the means of a Fast Fourier Transform, which reduces our computation time.

For our simulations, we take a level $\alpha=5 \%$ and a number of observations $n=100$. We choose $d_{0}$ and $Q$ both equal to 7 . The alternatives that we consider are the following ones:

$$
\begin{aligned}
& f_{a}(x)=a \sin (12 \pi x), \\
& g_{a}(x)=a(\sin (16 \pi x)+\sin (8 \pi x)), \\
& h_{a}(x)=a\left(\cos \left(\frac{16 \pi}{15} \cos (12 \pi x)\right)+\cos \left(\frac{8 \pi}{15} \cos (4 \pi x)\right)\right), \\
& j_{a}(x)=a \cos \left(\frac{16 \pi}{15} \cos (8 \pi x)\right) .
\end{aligned}
$$

We present in Table 3 the estimated powers for the tests based on $T_{p e r}$ and $T_{n p e r}$ under various alternatives. These powers are evaluated by 5000 simulations.

Comments.

As expected from our theoretical study, the above experimental results show that taking the periodicity assumption into account in the testing procedure improves the power of the test. This is all the more true if the number of significant Fourier coefficients (or harmonics) in the expansion of the periodic signal is large, as we can see for alternatives $h_{a}$ and $j_{a}$.

\section{Applichtions to LASER Vibrometry}

\subsection{Presentation of the laser vibrometry technique}

Let us consider a target vibrating under the effect of the vibrations of its motor for instance. If these vibrations are sinusoidal then the vibrations of a point number $m$ of the target can be written as $\gamma_{m} \cos \left(2 \pi F_{s} t\right)$, 
TABLE 1. Estimated powers for the tests based on $T_{p e r}$ and $T_{n p e r}$.

\begin{tabular}{|c|c|c|}
\hline \multicolumn{3}{|c|}{ Alternatives $f_{a}$} \\
\hline $\bar{a}$ & $T_{p e r}$ & $T_{\text {nper }}$ \\
\hline 1 & 1 & 1 \\
\hline 0.75 & 0.97 & 0.89 \\
\hline 0.5 & 0.61 & 0.42 \\
\hline 0.25 & 0.14 & 0.10 \\
\hline \multicolumn{3}{|c|}{ Alternatives $g_{a}$} \\
\hline$a$ & $T_{p e r}$ & $T_{n p e r}$ \\
\hline 1 & 1 & 0.97 \\
\hline 0.75 & 0.99 & 0.81 \\
\hline 0.5 & 0.89 & 0.43 \\
\hline 0.25 & 0.20 & 0.12 \\
\hline
\end{tabular}

\begin{tabular}{|ccc|}
\hline \multicolumn{3}{|c|}{ Alternatives $h_{a}$} \\
\hline$a$ & $T_{\text {per }}$ & $T_{\text {nper }}$ \\
\hline 1 & 1 & 0.23 \\
0.75 & 0.96 & 0.15 \\
0.5 & 0.56 & 0.09 \\
0.25 & 0.11 & 0.06 \\
\hline \multicolumn{3}{|c|}{ Alternatives $j_{a}$} \\
\hline$a$ & $T_{\text {per }}$ & $T_{\text {nper }}$ \\
\hline 1 & 0.99 & 0.02 \\
0.75 & 0.86 & 0.02 \\
0.5 & 0.38 & 0.02 \\
0.25 & 0.08 & 0.02 \\
\hline
\end{tabular}

where $\gamma_{m}$ is the amplitude of these vibrations and $F_{s}$ the vibration frequency (that is $1 / F_{s}$ is the vibration period). The identification of a target can be done by estimating the frequency $F_{s}$.

To do this, one uses a coherent laser emitting a continuous wave that can be written: $e(t)=\exp (2 i \pi c t / \lambda)$, where $c$ is the light speed and $\lambda$ is the laser wavelength (namely $1.5 \mu \mathrm{m}$ ). After emission of a continuous laser wave, reflection of it on the target, reception and demodulation, one receives a complex valued signal of the form:

$$
Y_{j}=f(j / n)+\sigma\left(\varepsilon_{1, j}+i \varepsilon_{2, j}\right) \text { for } j \in\{1, \ldots, n\},
$$

where $i^{2}=-1$, the $\varepsilon_{1, j}$ 's and the $\varepsilon_{2, j}$ 's are independent standard Gaussian random variables and

$$
f(t)=\sum_{m=1}^{M} a_{m} \exp \left[\frac{4 i \pi \gamma_{m}}{\lambda} \cos \left(2 \pi F_{s} t\right)\right],
$$

when the target consists of $M$ (which may be large: $M \approx 200$ ) punctual reflectors, $a_{m}$ being the amplitude of the signal reflected by the reflector number $m$.

A natural preliminary step to the target identification thus consists in testing from the received observations: " $f=0$ " against " $f \neq 0$ " which amounts to detecting the presence of any target. Of course, at that stage of detection we do not have any knowledge of the frequency $F_{s}$. We hence use the above testing method.

Then we could start the identification step which consists in estimating the frequency $F_{s}$ (see Lavielle and Lévy-Leduc [14] and Prenat [17] for more details on this point).

Let us now present a synthetic signal arising in laser vibrometry.

\subsection{Presentation of an example}

Figure 1a displays the imaginary part of $f(j / n)$ satisfying (17) for $j \in\{1, \ldots, 9000\}$ when $n=2^{18}, M=1$, $a_{1}=0.06, \gamma_{1}=35 \times 10^{-6}, \lambda=1.5 \times 10^{-6}$ and $F_{s}=48 \mathrm{~Hz}$. Figure $1 \mathrm{~b}$ displays the periodogram of $(f(j / n), j \in$ $\{1, \ldots, n\})$ defined by

$$
I_{f}(q)=\frac{1}{n}\left|\sum_{j=1}^{n} \mathrm{e}^{-\frac{2 i \pi q j}{n}} f\left(\frac{j}{n}\right)\right|^{2} \text { for } q \in\{0, \ldots, n-1\} .
$$

A zoom of this periodogram is presented in Figure 1c.

We can remark in this example that, with such a definition, $f$ is a periodic function with frequency $48 \mathrm{~Hz}$ with about 330 significant Fourier coefficients of positive index (or positive harmonics). 

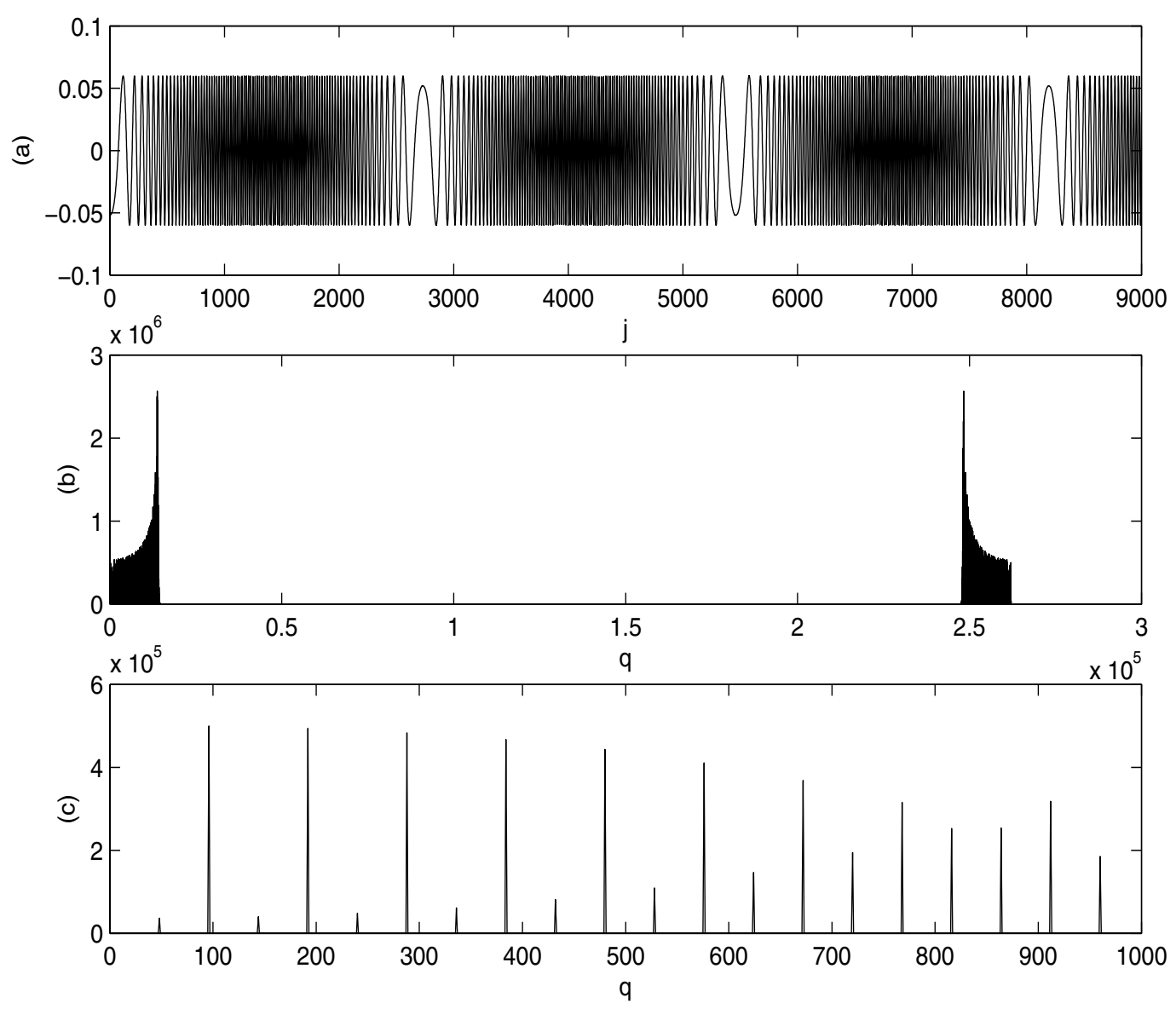

Figure 1. A synthetic signal $f$. (a) the imaginary part of $f$, (b) the periodogram of $f$, (c) zoom of (b).

Following Lavielle and Lévy-Leduc [14], when some noise $\varepsilon_{j}$ with variance $2 \sigma^{2}$ is added to $f(j / n)$, we characterize the signal to noise ratio by the so-called $S N R$ (in $\mathrm{dB}$ ) where

$$
S N R=10 \log \left(\sum_{m=1}^{M} a_{m}^{2} / 2 \sigma^{2}\right)
$$

Figure 2a displays the imaginary part of the $Y_{j}$ 's satisfying (16) when $f$ is the above deterministic signal and $S N R=-27 \mathrm{~dB}$ for $j \in\{1, \ldots, 9000\}$. In Figures $2 \mathrm{~b}$ and $2 \mathrm{c}$, one can see the corresponding periodogram defined by

$$
I_{Y}(q)=\frac{1}{n}\left|\sum_{j=1}^{n} \mathrm{e}^{-\frac{2 i \pi q j}{n}} Y_{j}\right|^{2}, \text { for } q \in\{0, \ldots, n-1\},
$$

and a zoom of it. We can notice in this example that, because of the high level of noise, the original signal and its harmonics cannot be visually detected. 

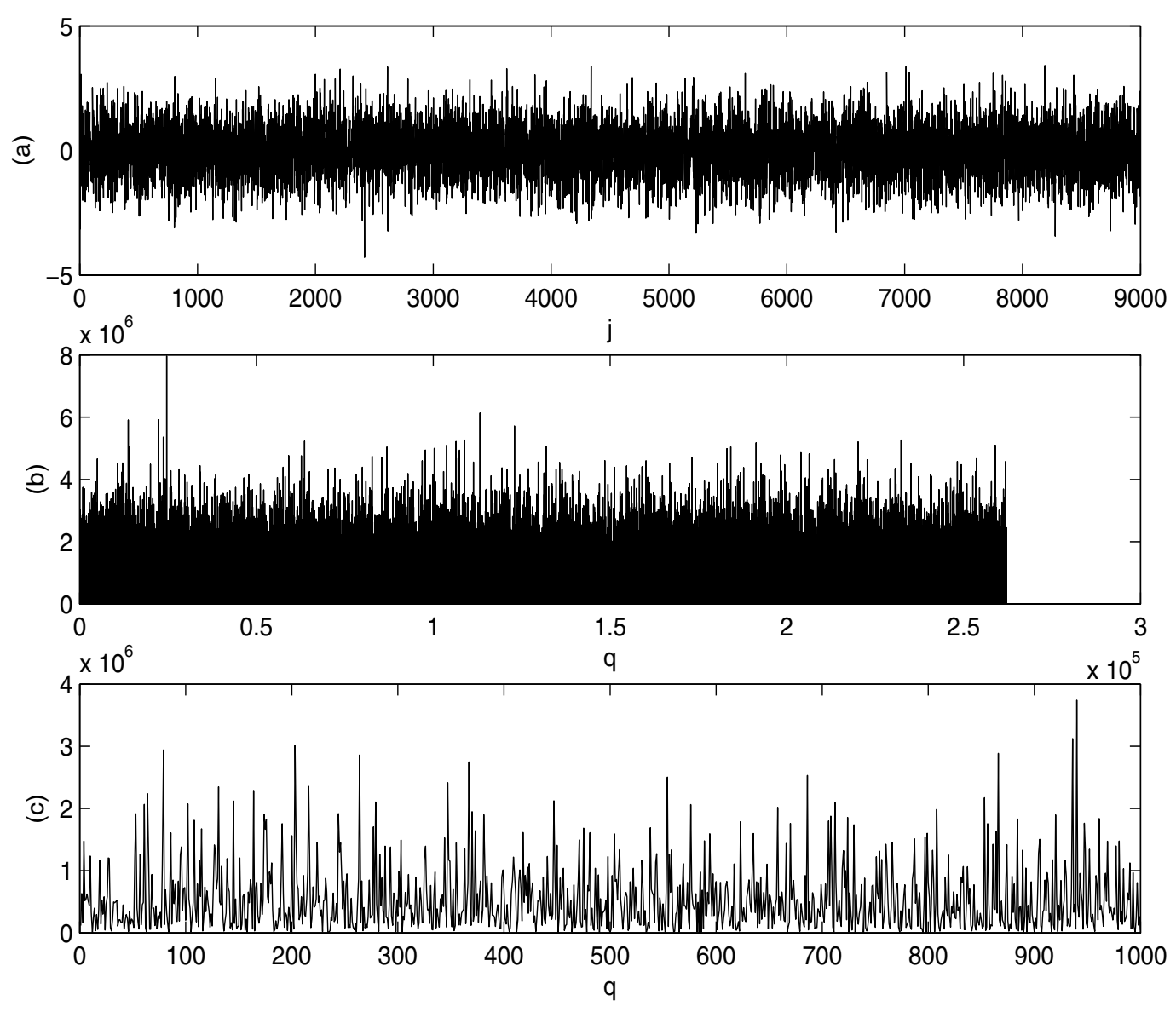

Figure 2. The observed signal $f+\varepsilon$. (a) the imaginary part of $f+\varepsilon$, (b) the periodogram of $f+\varepsilon,(\mathrm{c})$ zoom of (b).

\subsection{Application of our testing procedure}

Observing some $Y_{j}$ 's following the model (16) amounts in fact to observing two real data sets given by the imaginary and real parts of the $Y_{i}$ 's, which both satisfy (1). We can therefore use the procedure described in Section 2, at the price of slight changes in the definition of the test statistic $T_{\alpha}$. More precisely, we replace the norm $\|\cdot\|_{\mathbb{R}^{n}}$ by $\|\cdot\|_{\mathbb{C}^{n}}$, where $\|V\|_{\mathbb{C}^{n}}^{2}=n^{-1} \sum_{j=1}^{n}\left|V_{j}\right|^{2}$ for all $V=\left(V_{1}, \ldots, V_{n}\right)^{T} \in \mathbb{C}^{n}$, and we choose the collection $\left\{S_{m}, m \in \mathcal{M}\right\}$ as follows:

$$
\mathcal{M}=\left\{(q, D), q \in\{1, \ldots, Q\}, D \in\left\{2^{d}, d \in\left\{1, \ldots, d_{0}\right\}\right\}\right\},
$$

and for each $(q, D)$ in $\mathcal{M}, S_{(q, D)}$ is the linear space spanned by the vectors

$$
\left\{\left(\mathrm{e}^{2 i \pi q l / n}, \ldots, \mathrm{e}^{2 i \pi q l}\right)^{T}, l=-D, \ldots, D\right\} .
$$

We consider an alternative which corresponds to the signal (17) where $M=1, F_{s}=48 \mathrm{~Hz}, \gamma_{1}=35 \times 10^{-6}$, $\lambda=1.5 \times 10^{-6}$, with a noise such that $\sigma^{2}=1$. The computation of the test statistic is conducted in the same 
TABLE 2. Estimated powers of the test for various $S N R$.

\begin{tabular}{|c|c|c|c|c|c|c|c|c|c|c|c|}
\hline$S N R$ & -34 & -33 & -32 & -31 & -30 & -29 & -28 & -27 & -26 & -25 & -24 \\
\hline Power & 0.05 & 0.07 & 0.10 & 0.13 & 0.18 & 0.29 & 0.45 & 0.67 & 0.85 & 0.97 & 0.99 \\
\hline
\end{tabular}

way as in Section 3: with very similar calculations, we actually obtain that when $2^{d_{0}+1} Q<n$,

$$
\left\|\Pi_{(q, D)}(Y)\right\|_{\mathbb{C}^{n}}^{2}=\frac{1}{n^{2}} \sum_{l=-D}^{D}\left|\sum_{j=1}^{n} \mathrm{e}^{-2 i \pi l j q / n} Y_{j}\right|^{2}
$$

For a number of observations $n=2^{18}$, we have chosen $Q=50$ and $d_{0}=10$. We take a level of significance $\alpha=5 \%$. The quantiles $\bar{F}_{D_{m}, n-D_{m}}^{-1}\left(u_{\alpha}\right)$ are estimated by 40000 simulations. We use 20000 simulations for the estimation of $\bar{F}_{D_{m}, n-D_{m}}^{-1}(u)$ for $u$ varying on a regular grid of ]0, $\alpha$ [ with mesh 1/20000 and 20000 for the estimation of the probabilities

$$
\mathbb{P}_{0}\left(\sup _{m \in \mathcal{M}}\left\{\frac{\left(n-D_{m}\right)\left\|\Pi_{m}(Y)\right\|_{\mathbb{R}^{n}}^{2}}{D_{m}\left\|Y-\Pi_{m}(Y)\right\|_{\mathbb{R}^{n}}^{2}}-\bar{F}_{D_{m}, n-D_{m}}^{-1}(u)\right\}>0\right)
$$

Table 4.3 presents the estimated powers for various $S N R$, namely $-34,-33, \ldots,-24 \mathrm{~dB}$.

\section{Comments.}

Since the minimal $S N R$ needed to perform target detection is directly linked to the power that the laser has to emit, a $S N R$ about $-24 \mathrm{~dB}$ is reasonable for the application that we have in view. The results summarized in Table 4.3 show that, at such a signal to noise ratio, our test is powerful. The classical tests used in periodic signal detection, such as those developed in the books of Brockwell and Davis [4] or Quinn and Hannan [18], essentially use a parametric approach. As noticed in [6] on page 298, they are particularly adapted to the detection of periodic signals having a small number of harmonics such as sinusoids or trigonometric polynomials with small degrees, which is not the case of the signals of our interest. Therefore, we can think that they will not be effective in our context. This is furthermore confirmed by an experimental study: by implementing the test proposed by [4], we found for a $S N R$ of $-24 \mathrm{~dB}$ a power of 0.06 .

\section{Conclusion And prospects}

In this paper, we propose a testing procedure to detect the presence of a periodic signal corrupted by a Gaussian noise. The originality of our approach as compared to the other works on adaptive signal detection lies in its sensitivity to the periodicity of the signal. This leads to significant improvements on the existing methods from both theoretical and practical points of view.

There are several ways in which the results of this paper can be extended. Indeed, it can be interesting to perform the same study but in a framework where the errors are not necessarily Gaussian, so that it can handle a larger field of real world applications. With a view to the laser vibrometry issue, it is also interesting to extend the present testing method to the case where the regression function is not a unique periodic function but a sum of several periodic functions with different periods, and to propose in parallel a criterion to estimate the number of corresponding periodic signals. 


\section{Proofs}

\subsection{Proof of Theorem 1}

The idea of the proof is to construct, for each Sobolev ball $\mathcal{S}_{k}(s, R)$, a sphere $\Omega$ of maximal radius such that $\beta(\Omega) \geq \delta$ and $\Omega \subset \mathcal{S}_{k}(s, R)$. Let $k \in\{1, \ldots, n\}, s \in \mathbb{N}^{*}$ and $R>0$. As Ingster [12] suggests, we introduce a compactly supported and smooth enough function $\phi$ : let $\phi$ be some function in $\mathcal{C}^{\infty}(\mathbb{R})$ supported by $] 0,1[$ and positive on its support (we can take for example the function defined on $] 0,1\left[\right.$ by $\phi(x)=\exp \left(-1 / x^{2}-1 /(x-1)^{2}\right)$ ). For all $D \in \mathbb{N}^{*}$, we consider

$$
\widetilde{S_{D}}=\left\{f, f(x)=\sum_{l=1}^{D} \beta_{l} \phi_{D}\left(\frac{n D x}{k}-l+1\right), \beta_{l} \in \mathbb{R}\right\},
$$

where $\phi_{D}$ is the function in $\mathcal{C}^{\infty}(\mathbb{R})$ which is periodic with period $D$ and which agrees with $\phi$ on $\left.] 0, D\right]$.

Let

$$
\kappa_{1}=1 \wedge \frac{\int_{0}^{1} \phi^{2}(x) \mathrm{d} x}{16\left\|\phi \phi^{\prime}\right\|_{\infty}} \text { and } \kappa_{2}=\frac{1}{\sqrt{6}}\left(\frac{\int_{0}^{1} \phi^{2}(x) \mathrm{d} x}{4\left\|\phi \phi^{\prime}\right\|_{\infty}+\int_{0}^{1} \phi^{2}(x) \mathrm{d} x}\right) .
$$

The following paragraph is devoted to a study of the spheres of the form $\left\{f \in \widetilde{S_{D}},\|f\|_{n}=\rho\right\}$.

- A lower bound for $\tilde{\rho}\left(\widetilde{S_{D}}, \alpha, \delta, \sigma\right)$

Proposition 4. Assume that $\kappa_{1}^{-1} \leq k \leq n$. For all $D \in\left\{1, \ldots,\left[\kappa_{1} k\right]\right\}$ and for all $\rho^{2} \leq \kappa_{2}(1-\alpha-\delta) \sqrt{D} \sigma^{2} / n$,

$$
\beta\left(\left\{f \in \widetilde{S_{D}},\|f\|_{n}=\rho\right\}\right) \geq \delta .
$$

Comments:

This proposition gives a result in terms of minimax separation rates. Indeed, it is easy to see that it implies the lower bound:

$$
\tilde{\rho}^{2}\left(\widetilde{S_{D}}, \alpha, \delta, \sigma\right) \geq \kappa_{2}(1-\alpha-\delta) \sqrt{D} \sigma^{2} / n
$$

Proof: By homogeneity, we can only consider the case where $\sigma=1$. Let $D \in\left\{1, \ldots,\left[\kappa_{1} k\right]\right\}$ and for $\rho>0$

$$
\widetilde{S_{D}}[\rho]=\left\{f \in \widetilde{S_{D}},\|f\|_{n}=\rho\right\}
$$

Recall that $\mathbb{P}_{f}$ denotes the distribution of the observation $Y=\left(Y_{1}, \ldots, Y_{n}\right)$ defined by (1).

The proof of Proposition 4 is based on the following classical bound on the error of tests : in the notation of Baraud [1], if

- $\mu_{\rho}$ is a probability measure on $\widetilde{S_{D}}[\rho]$;

- $P_{\mu_{\rho}}=\int \mathbb{P}_{f} \mathrm{~d} \mu_{\rho}(f)$;

- $L_{\mu_{\rho}}(y)=\frac{\mathrm{d} P_{\mu_{\rho}}}{\mathrm{d} \mathbb{P}_{0}}(y)$

- $\mathbb{E}_{0}$ is the expectation with respect to $\mathbb{P}_{0}$,

then

$$
\beta\left(\widetilde{S_{D}}[\rho]\right) \geq 1-\alpha-\frac{1}{2}\left(\mathbb{E}_{0}\left[L_{\mu_{\rho}}^{2}(Y)\right]-1\right)^{1 / 2} .
$$

The main difficulty then consists in finding some $\mu_{\rho}$ such that for all $\rho^{2} \leq \kappa_{2}(1-\alpha-\delta) \sqrt{D} \sigma^{2} / n$,

$$
\mathbb{E}_{0}\left[L_{\mu_{\rho}}^{2}(Y)\right] \leq 1+4(1-\alpha-\delta)^{2}
$$

Let $\xi=\left(\xi_{1}, \ldots, \xi_{D}\right)$ be a sequence of Rademacher random variables that is a sequence of i.i.d. random variables taking the values +1 or -1 with probability $1 / 2$. 
Let $f_{\xi}(x)=\lambda \sum_{l=1}^{D} \xi_{l} \phi_{D}(n D x / k-l+1)$ where $\lambda$ is chosen such that $\left\|f_{\xi}\right\|_{n}=\rho$. By the properties of the support and the period of $\phi_{D}$ :

$$
\begin{aligned}
\left\|f_{\xi}\right\|_{n}^{2} & =\frac{\lambda^{2}}{n}[n / k] \sum_{j=1}^{k}\left(\sum_{l=1}^{D} \xi_{l} \phi_{D}\left(\frac{D j}{k}-l+1\right)\right)^{2}+\frac{\lambda^{2}}{n} \sum_{j=1}^{n-[n / k] k}\left(\sum_{l=1}^{D} \xi_{l} \phi_{D}\left(\frac{D j}{k}-l+1\right)\right)^{2} \\
& =\frac{\lambda^{2}}{n}[n / k] \sum_{l=1}^{D} \sum_{j \in A_{l, D}} \phi_{D}^{2}\left(\frac{D j}{k}-l+1\right)+\frac{\lambda^{2}}{n} \sum_{l=1}^{D} \sum_{j \in A_{l, D}^{\prime}} \phi_{D}^{2}\left(\frac{D j}{k}-l+1\right) \\
& =\frac{\lambda^{2}}{n} \sum_{l=1}^{D}\left([n / k] \sum_{j \in A_{l, D}} \phi_{D}^{2}\left(\frac{D j}{k}-l+1\right)+\sum_{j \in A_{l, D}^{\prime}} \phi_{D}^{2}\left(\frac{D j}{k}-l+1\right)\right)
\end{aligned}
$$

where $A_{l, D}=\{j \in] \frac{(l-1) k}{D}, \frac{l k}{D}[\}$ and $A_{l, D}^{\prime}=A_{l, D} \cap\{1, \ldots, n-[n / k] k\}$.

Taking

$$
\lambda^{2}=n \rho^{2}\left[\sum_{l=1}^{D}\left([n / k] \sum_{j \in A_{l, D}} \phi_{D}^{2}\left(\frac{D j}{k}-l+1\right)+\sum_{j \in A_{l, D}^{\prime}} \phi_{D}^{2}\left(\frac{D j}{k}-l+1\right)\right)\right]^{-1}
$$

implies that $f_{\xi} \in \widetilde{S_{D}}[\rho]$, which allows us to take $\mu_{\rho}$ as the distribution of $f_{\xi}$. With such a choice for $\mu_{\rho}$, we have

$$
\begin{aligned}
L_{\mu_{\rho}}(y) & =\mathbb{E}_{\xi}\left(\exp \left\{-\frac{1}{2} \sum_{j=1}^{n}\left[y_{j}-\lambda \sum_{l=1}^{D} \xi_{l} \phi_{D}\left(\frac{D j}{k}-l+1\right)\right]^{2}+\frac{1}{2} \sum_{j=1}^{n} y_{j}^{2}\right\}\right) \\
& =\mathrm{e}^{-\frac{n \rho^{2}}{2}} \mathbb{E}_{\xi}\left(\exp \left\{\lambda \sum_{j=1}^{n} y_{j} \sum_{l=1}^{D} \xi_{l} \phi_{D}\left(\frac{D j}{k}-l+1\right)\right\}\right) \\
& =\mathrm{e}^{-\frac{n \rho^{2}}{2}} \mathbb{E}_{\xi}\left(\exp \left\{\begin{array}{c}
\lambda \sum_{l=1}^{D} \xi_{l} \sum_{m=0}^{[n / k]-1} \sum_{j=m k+1}^{m k+k} y_{j} \phi_{D}\left(\frac{D j}{k}-l+1\right) \\
+\lambda \sum_{l=1}^{D} \xi_{l} \sum_{j=[n / k] k+1}^{n} y_{j} \phi_{D}\left(\frac{D j}{k}-l+1\right)
\end{array}\right\}\right) .
\end{aligned}
$$

From the properties of the support of $\phi_{D}$, we get

$$
L_{\mu_{\rho}}(y)=\mathrm{e}^{-\frac{n \rho^{2}}{2}} \mathbb{E}_{\xi}\left(\exp \left\{\begin{array}{c}
\lambda \sum_{l=1}^{D} \xi_{l} \sum_{m=0}^{[n / k]-1} \sum_{j \in A_{m, l, D}} y_{j} \phi_{D}\left(\frac{D j}{k}-l+1\right) \\
+\lambda \sum_{l=1}^{D} \xi_{l} \sum_{j \in\{[n / k] k+1, \ldots, n\} \cap A_{[n / k], l, D}} y_{j} \phi_{D}\left(\frac{D j}{k}-l+1\right)
\end{array}\right\}\right),
$$

where

and by independence of the $\xi_{l}$ 's, we have

$$
A_{m, l, D}=\{j \in] m k+\frac{(l-1) k}{D}, m k+\frac{l k}{D}[\}
$$

$$
\mathbb{E}_{0}\left(L_{\mu_{\rho}}^{2}(Y)\right)=\mathrm{e}^{-n \rho^{2}} \mathbb{E}_{0}\left[\prod_{l=1}^{D} \cosh ^{2}\left(\begin{array}{c}
\lambda \sum_{m=0}^{[n / k]-1} \sum_{j \in A_{m, l, D}} Y_{j} \phi_{D}\left(\frac{D j}{k}-l+1\right) \\
+\lambda \sum_{j \in\{[n / k] k+1, \ldots, n\} \cap A_{[n / k], l, D}} Y_{j} \phi_{D}\left(\frac{D j}{k}-l+1\right)
\end{array}\right)\right] .
$$


Let

$$
B_{l}=\lambda \sum_{m=0}^{[n / k]-1} \sum_{j \in A_{m, l, D}} Y_{j} \phi_{D}\left(\frac{D j}{k}-l+1\right)+\lambda \sum_{j \in\{[n / k] k+1, \ldots, n\} \cap A_{[n / k], l, D}} Y_{j} \phi_{D}\left(\frac{D j}{k}-l+1\right) .
$$

Under the null hypothesis, the $Y_{j}$ 's are independent Gaussian random variables with mean 0 and variance 1 . Hence, the $B_{l}$ 's are independent Gaussian random variables with mean 0 and variance $\lambda^{2} \Sigma_{l}$ where

$$
\Sigma_{l}=\sum_{m=0}^{[n / k]-1} \sum_{j \in A_{m, l, D}} \phi_{D}^{2}\left(\frac{D j}{k}-l+1\right)+\sum_{j \in\{[n / k] k+1, \ldots, n\} \cap A_{[n / k], l, D}} \phi_{D}^{2}\left(\frac{D j}{k}-l+1\right) .
$$

By periodicity of $\phi_{D}$, we have that

$$
\Sigma_{l}=[n / k] \sum_{j \in A_{l, D}} \phi_{D}^{2}\left(\frac{D j}{k}-l+1\right)+\sum_{j \in A_{l, D}^{\prime}} \phi_{D}^{2}\left(\frac{D j}{k}-l+1\right) .
$$

This implies via (19) that

$$
\lambda^{2}=n \rho^{2}\left(\sum_{l=1}^{D} \Sigma_{l}\right)^{-1}
$$

and we get

$$
\begin{aligned}
\mathbb{E}_{0}\left(L_{\mu_{\rho}}^{2}(Y)\right) & =\mathrm{e}^{-n \rho^{2}} \prod_{l=1}^{D} \mathrm{e}^{\lambda^{2} \Sigma_{l}} \cosh \left(\lambda^{2} \Sigma_{l}\right) \\
& \leq \mathrm{e}^{-n \rho^{2}} \exp \left(\lambda^{2} \sum_{l=1}^{D} \Sigma_{l}\right) \exp \left(\frac{\lambda^{4}}{2} \sum_{l=1}^{D} \Sigma_{l}^{2}\right) \\
& \leq \exp \left(\frac{\lambda^{4}}{2} \sum_{l=1}^{D} \Sigma_{l}^{2}\right) \leq \exp \left(\frac{n^{2} \rho^{4}}{2} \frac{\sum_{l=1}^{D} \Sigma_{l}^{2}}{\left(\sum_{l=1}^{D} \Sigma_{l}\right)^{2}}\right) .
\end{aligned}
$$

Let us turn to an evaluation of $\Sigma_{l}$, which is the object of the following lemma.

Lemma 1. Let $1 \leq D \leq k \leq n$ and let for $l \in\{1, \ldots, D\}, A_{l, D}=\{j \in] \frac{(l-1) k}{D}, \frac{l k}{D}[\}$, and $A_{l, D}^{\prime}=A_{l, D} \cap$ $\{1, \ldots, n-[n / k] k\}$. The quantity $\Sigma_{l}$ defined by

$$
\Sigma_{l}=[n / k] \sum_{j \in A_{l, D}} \phi_{D}^{2}\left(\frac{D j}{k}-l+1\right)+\sum_{j \in A_{l, D}^{\prime}} \phi_{D}^{2}\left(\frac{D j}{k}-l+1\right)
$$

satisfies the two following inequalities:

$$
\Sigma_{l} \leq 2[n / k] \frac{k}{D}\left(4\left\|\phi \phi^{\prime}\right\|_{\infty}+\int_{0}^{1} \phi^{2}(x) \mathrm{d} x\right),
$$

and if $8\left\|\phi \phi^{\prime}\right\|_{\infty} \leq(k / 2 D) \int_{0}^{1} \phi^{2}(x) \mathrm{d} x$ (that is if $D \leq \kappa_{1} k$ ),

$$
\Sigma_{l} \geq[n / k] \frac{k}{2 D} \int_{0}^{1} \phi^{2}(x) \mathrm{d} x .
$$


From Lemma 1, we deduce that for all $D \in\left\{1, \ldots,\left[\kappa_{1} k\right]\right\}$,

$$
\sum_{l=1}^{D} \Sigma_{l}^{2}\left(\sum_{l=1}^{D} \Sigma_{l}\right)^{-2} \leq \frac{8}{3 \kappa_{2}^{2} D}
$$

Hence, as soon as

$$
\rho^{2} \leq \kappa_{2}(1-\alpha-\delta) \frac{\sqrt{D}}{n} \leq \kappa_{2} \frac{\sqrt{3}}{2} \frac{\sqrt{\log \left(1+4(1-\alpha-\delta)^{2}\right) D}}{n},
$$

from (20), we derive that

$$
\mathbb{E}_{0}\left(L_{\mu_{\rho}}^{2}(Y)\right) \leq 1+4(1-\alpha-\delta)^{2}
$$

and the result follows.

Proof of Lemma 1: To simplify the expressions, we set $x_{j, l}=D j / k-l+1$. Let us first give a lower bound for $\Sigma_{l}$. It is obvious that

$$
\begin{aligned}
& \Sigma_{l} \geq[n / k] \sum_{j=[(l-1) k / D]}^{[l k / D]} \phi_{D}^{2}\left(x_{j, l}\right)-[n / k] \phi_{D}^{2}\left(x_{[(l-1) k / D], l}\right) \\
& \geq[n / k] \frac{k}{D} \sum_{j=[(l-1) k / D]}^{[l k / D]}\left(\frac{D}{k} \phi_{D}^{2}\left(x_{j, l}\right)-\int_{x_{j, l}}^{x_{j+1, l}} \phi_{D}^{2}(x) \mathrm{d} x\right) \\
& \quad+[n / k] \frac{k}{D} \sum_{j=[(l-1) k / D]}^{[l k / D]} \int_{x_{j, l}}^{x_{j+1, l}} \phi_{D}^{2}(x) \mathrm{d} x-[n / k] \phi_{D}^{2}\left(x_{[(l-1) k / D], l}\right) .
\end{aligned}
$$

Since $\phi_{D}(0)=0$, this implies that

$$
\begin{aligned}
\Sigma_{l} \geq & {[n / k] \frac{k}{D} \int_{0}^{1} \phi_{D}^{2}(x) \mathrm{d} x-[n / k]\left(\phi_{D}^{2}\left(x_{[(l-1) k / D], l}\right)-\phi_{D}^{2}(0)\right) } \\
& -[n / k] \frac{k}{D} \sum_{j=[(l-1) k / D]}^{[l k / D]} \int_{x_{j, l}}^{x_{j+1, l}}\left|\phi_{D}^{2}\left(x_{j, l}\right)-\phi_{D}^{2}(x)\right| \mathrm{d} x,
\end{aligned}
$$

which allows us to use the Lipshitz properties of $\phi_{D}$. We thus obtain

$$
\begin{aligned}
\Sigma_{l} & \geq[n / k] \frac{k}{D} \int_{0}^{1} \phi_{D}^{2}(x) \mathrm{d} x-[n / k] 2\left\|\phi_{D} \phi_{D}^{\prime}\right\|_{\infty}\left|x_{[(l-1) k / D], l}\right|-2\left\|\phi_{D} \phi_{D}^{\prime}\right\|_{\infty}[n / k] \frac{k}{D}\left(\frac{k}{D}+2\right)\left(\frac{D}{k}\right)^{2} \\
& \geq[n / k]\left(\frac{k}{D} \int_{0}^{1} \phi_{D}^{2}(x) \mathrm{d} x-8\left\|\phi_{D} \phi_{D}^{\prime}\right\|_{\infty}\right) .
\end{aligned}
$$

Since $\phi_{D}$ agrees with $\phi$ on $] 0,1\left[, \Sigma_{l} \geq[n / k]\left(\frac{k}{D} \int_{0}^{1} \phi^{2}(x) \mathrm{d} x-8\left\|\phi \phi^{\prime}\right\|_{\infty}\right)\right.$. So if $8\left\|\phi \phi^{\prime}\right\|_{\infty} \leq(k / 2 D) \int_{0}^{1} \phi^{2}(x) \mathrm{d} x$,

$$
\Sigma_{l} \geq[n / k] \frac{k}{2 D} \int_{0}^{1} \phi^{2}(x) \mathrm{d} x
$$


The upper bound is obtained by similar arguments. Since $D \leq k \leq n$,

$$
\begin{aligned}
\Sigma_{l} \leq & 2[n / k] \sum_{j=[(l-1) k / D]+1}^{[l k / D]} \phi_{D}^{2}\left(x_{j, l}\right) \\
\leq & 2[n / k] \frac{k}{D} \sum_{j=[(l-1) k / D]+1}^{[l k / D]-1}\left(\frac{D}{k} \phi_{D}^{2}\left(x_{j, l}\right)-\int_{x_{j, l}}^{x_{j+1, l}} \phi_{D}^{2}(x) \mathrm{d} x\right) \\
& +2[n / k] \frac{k}{D} \sum_{j=[(l-1) k / D]+1}^{[l k / D]-1} \int_{x_{j, l}}^{x_{j+1, l}} \phi_{D}^{2}(x) \mathrm{d} x+2[n / k] \phi_{D}^{2}\left(x_{[l k / D], l}\right) .
\end{aligned}
$$

The fact that $\phi_{D}(1)=0$ leads as above to

$$
\begin{aligned}
\Sigma_{l} \leq & 2[n / k] \frac{k}{D} \sum_{j=[(l-1) k / D]+1}^{[l k / D]-1} \int_{x_{j, l}}^{x_{j+1, l}}\left(\phi_{D}^{2}\left(x_{j, l}\right)-\phi_{D}^{2}(x)\right) \mathrm{d} x \\
& +2[n / k] \frac{k}{D} \int_{0}^{1} \phi_{D}^{2}(x) \mathrm{d} x+2[n / k]\left(\phi_{D}^{2}\left(x_{[l k / D]}\right)-\phi_{D}^{2}(1)\right) \\
& \leq 4[n / k] \frac{k}{D} \sum_{j=[(l-1) k / D]+1}^{[l k / D]-1}\left\|\phi_{D} \phi_{D}^{\prime}\right\|_{\infty}\left(\frac{D}{k}\right)^{2}+2[n / k] \frac{k}{D} \int_{0}^{1} \phi_{D}^{2}(x) \mathrm{d} x+4[n / k]\left\|\phi_{D} \phi_{D}^{\prime}\right\|_{\infty} \frac{D}{k} \\
\leq & 2[n / k] \frac{k}{D}\left(4\left\|\phi \phi^{\prime}\right\|_{\infty}+\int_{0}^{1} \phi^{2}(x) \mathrm{d} x\right),
\end{aligned}
$$

which completes the proof.

\section{- A lower bound for $\tilde{\rho}\left(\mathcal{S}_{k}(s, R), \alpha, \delta, \sigma\right)$}

Let $k$ satisfy $\kappa_{1}^{-1} \leq k \leq n / 2$. For all $D \in\left\{1, \ldots,\left[\kappa_{1} k\right]\right\}$, we introduce

$$
r_{D, k, s}^{2}=\frac{1}{4} \frac{\int_{0}^{1} \phi^{2}(x) \mathrm{d} x}{\int_{0}^{1} \phi^{(s)^{2}}(x) \mathrm{d} x} R^{2} D^{-2 s}\left(\frac{k}{n}\right)^{2 s} \wedge \kappa_{2}(1-\alpha-\delta) \frac{\sqrt{D} \sigma^{2}}{n},
$$

and we aim here to obtain the lower bound:

$$
\tilde{\rho}^{2}\left(\mathcal{S}_{k}(s, R), \alpha, \delta, \sigma\right) \geq \sup _{D \in\left\{1, \ldots,\left[\kappa_{1} k\right]\right\}} r_{D, k, s}^{2} .
$$

The fact that $r_{D, k, s}^{2} \leq \kappa_{2}(1-\alpha-\delta) \sqrt{D} \sigma^{2} / n$ implies by Proposition 4 that

$$
\beta\left(\left\{f \in \widetilde{S_{D}},\|f\|_{n}=r_{D, k, s}\right\}\right) \geq \delta .
$$

Hence, we only need to prove that for every $D \in\left\{1, \ldots,\left[\kappa_{1} k\right]\right\}$,

$$
\left\{f \in \widetilde{S_{D}},\|f\|_{n}=r_{D, k, s}\right\} \subset\left\{f \in \mathcal{S}_{k}(s, R),\|f\|_{n} \geq r_{D, k, s}\right\},
$$

to ensure that

$$
\beta\left(\left\{f \in \mathcal{S}_{k}(s, R),\|f\|_{n} \geq r_{D, k, s}\right\}\right) \geq \delta,
$$

for all $D \in\left\{1, \ldots,\left[\kappa_{1} k\right]\right\}$. The expected lower bound for $\tilde{\rho}^{2}\left(\mathcal{S}_{k}(s, R), \alpha, \delta, \sigma\right)$ will then directly follow. 
So, fixing $D \in\left\{1, \ldots,\left[\kappa_{1} k\right]\right\}$, let us take $f$ in $\widetilde{S_{D}}$ with

$$
f(x)=\sum_{l=1}^{D} \beta_{l} \phi_{D}\left(\frac{D n x}{k}-l+1\right),
$$

and prove that $\|f\|_{n}=r_{D, k, s}$ implies that $f$ belongs to $\mathcal{S}_{k}(s, R)$. It is easy to see that $f$ belongs to $\mathcal{C}^{s}([0,1])$ and that $f$ is periodic with period $k / n$. Furthermore, by the properties of the support of $\phi_{D}$,

$$
\begin{aligned}
\frac{n}{k} \int_{0}^{k / n} f^{(s)^{2}}(x) \mathrm{d} x & =\frac{n}{k} \int_{0}^{k / n}\left(\sum_{l=1}^{D} \beta_{l}^{2}\left(\frac{D n}{k}\right)^{2 s} \phi_{D}^{(s)^{2}}\left(\frac{D n x}{k}-l+1\right)\right) \mathrm{d} x \\
& =\left(\frac{D n}{k}\right)^{2 s} \frac{1}{D} \sum_{l=1}^{D} \beta_{l}^{2} \int_{0}^{1} \phi^{(s)^{2}}(y) \mathrm{d} y .
\end{aligned}
$$

From the assumptions on the period and the support of $\phi_{D}$, we can see that:

$$
\|f\|_{n}^{2}=\frac{1}{n} \sum_{l=1}^{D} \beta_{l}^{2}\left(\left[\frac{n}{k}\right] \sum_{j \in A_{l, D}} \phi_{D}^{2}\left(\frac{D j}{k}-l+1\right)+\sum_{j \in A_{l, D}^{\prime}} \phi_{D}^{2}\left(\frac{D j}{k}-l+1\right)\right)
$$

with

and

$$
A_{l, D}=\{j \in] \frac{(l-1) k}{D}, \frac{l k}{D}[\}
$$

Lemma 1 then leads to the inequality:

$$
A_{l, D}^{\prime}=A_{l, D} \cap\{1, \ldots, n-[n / k] k\} .
$$

$$
\|f\|_{n}^{2} \geq \frac{1}{4 D} \sum_{l=1}^{D} \beta_{l}^{2} \int_{0}^{1} \phi^{2}(x) \mathrm{d} x .
$$

Hence, if

we have that

$$
\|f\|_{n}^{2}=r_{D, k, s}^{2} \leq \frac{1}{4} \frac{\int_{0}^{1} \phi^{2}(x) \mathrm{d} x}{\int_{0}^{1} \phi^{(s)^{2}}(x) \mathrm{d} x} R^{2}\left(\frac{k}{n}\right)^{2 s} D^{-2 s}
$$

$$
\frac{1}{D} \sum_{l=1}^{D} \beta_{l}^{2} \leq \frac{R^{2}\left(\frac{k}{n}\right)^{2 s} D^{-2 s}}{\int_{0}^{1} \phi^{(s)^{2}}(x) \mathrm{d} x} .
$$

From (21), we finally deduce that

$$
\frac{n}{k} \int_{0}^{k / n} f^{(s)^{2}}(x) \mathrm{d} x \leq R^{2}
$$

so, $f \in \mathcal{S}_{k}(s, R)$. This concludes the proof of Theorem 1 .

\subsection{Proof of Proposition 1}

Let $\bar{f}=(f(1 / n), \ldots, f(1))^{T}$. As in Theorem 2 from Baraud [1], by using some deviations inequalities due to Birgé [3] and Laurent and Massart [13], since the dimension of $S_{D_{k}^{*}}$ is smaller than $D_{k}^{*}$, we can prove that for all $f$ satisfying:

$$
\|f\|_{n}^{2} \geq\left\{\left\|\bar{f}-\Pi_{S_{D_{k}^{*}}}(\bar{f})\right\|_{\mathbb{R}^{n}}^{2}+2(\sqrt{5}+4) \log \left(\frac{1}{\alpha \delta}\right) \frac{\sqrt{D_{k}^{*}}}{n} \sigma^{2}\right\}
$$


then

$$
\mathbb{P}_{f}\left(\Phi_{\alpha}^{*}=0\right) \leq \delta
$$

The final result is obtained by computing an upper bound for $\left\|\bar{f}-\Pi_{S_{D_{k}^{*}}}(\bar{f})\right\|_{\mathbb{R}^{n}}^{2}$ when $f$ belongs to $\mathcal{S}_{k}(s, R)$. This can be derived via the following lemma.

Lemma 2. Let $d_{n}$ be the distance associated with the norm $\|\cdot\|_{\mathbb{R}^{n}}$ and for $D \in \mathbb{N}^{*}$, consider $S_{D}$ defined by (6). Let $f$ be some periodic function with period $k / n$ such that for all $x \in[0,1], f(x)=\sum_{l \geq 1} \beta_{l}(f) \varphi_{k / n, l}(x)$. If

$$
(2 \pi)^{2 s} \sum_{l \geq 2}[l / 2]^{2 s} \beta_{l}^{2}(f) \leq R^{2}\left(\frac{k}{n}\right)^{2 s},
$$

then, there exists some absolute constant $C>0$ such that, setting $\bar{f}=(f(1 / n), \ldots, f(1))^{T}$ :

$$
d_{n}^{2}\left(\bar{f}, S_{D}\right) \leq C\left(R^{2}\left(\frac{k}{n}\right)^{2 s} D^{-2 s}+\frac{R^{2} k}{n^{2 s}}\right) .
$$

The proof of Lemma 2 is postponed to the end of the section.

Assume that $f$ belongs to $\mathcal{S}_{k}(s, R)$. Then $f$ satisfies the assumptions of Lemma 2 and we deduce from Lemma 2 and (22) that there exists some positive constant $c(\alpha, \delta)$ such that if $f$ satisfies

$$
\begin{gathered}
\|f\|_{n}^{2} \geq c(\alpha, \delta)\left\{R^{2}\left(\frac{k}{n}\right)^{2 s} D_{k}^{*-2 s}+\frac{R^{2} k}{n^{2 s}}+\frac{\sqrt{D_{k}^{*}}}{n} \sigma^{2}\right\}, \\
\mathbb{P}_{f}\left(\Phi_{\alpha}^{*}=0\right) \leq \delta .
\end{gathered}
$$

Recalling that $D_{k}^{*}=D^{*} \wedge k$ where $D^{*}$ is the smallest integer at least equal to $\left(R^{2}(k / n)^{2 s} n / \sigma^{2}\right)^{2 /(1+4 s)}$, we now distinguish three cases.

If $D^{*}=1$, then $\left(R^{2}(k / n)^{2 s} n / \sigma^{2}\right)^{2 /(1+4 s)} \leq 1$ and $D_{k}^{*}=1$. Hence

$$
R^{2}\left(\frac{k}{n}\right)^{2 s} D_{k}^{*-2 s}+\frac{R^{2} k}{n^{2 s}}+\frac{\sqrt{D_{k}^{*}}}{n} \sigma^{2} \leq 3 \frac{\sigma^{2}}{n} .
$$

If $D^{*}>k,\left(R^{2}(k / n)^{2 s} n / \sigma^{2}\right)^{2 /(1+4 s)} \geq k$ and $D_{k}^{*}=k$. This implies that

$$
R^{2}\left(\frac{k}{n}\right)^{2 s} D_{k}^{*-2 s}+\frac{R^{2} k}{n^{2 s}}+\frac{\sqrt{D_{k}^{*}}}{n} \sigma^{2} \leq 3 \frac{R^{2} k}{n^{2 s}}
$$

Finally, if $2 \leq D^{*} \leq k$, since $D^{*}-1<\left(R^{2}(k / n)^{2 s} n / \sigma^{2}\right)^{2 /(1+4 s)} \leq D^{*}$ and $D^{*} \leq 2\left(D^{*}-1\right)$, we obtain that

$$
R^{2}\left(\frac{k}{n}\right)^{2 s} D_{k}^{*-2 s}+\frac{R^{2} k}{n^{2 s}}+\frac{\sqrt{D_{k}^{*}}}{n} \sigma^{2} \leq(1+\sqrt{2}) R^{\frac{2}{1+4 s}}\left(\frac{k}{n}\right)^{\frac{2 s}{1+4 s}}\left(\frac{\sigma^{2}}{n}\right)^{\frac{4 s}{4 s+1}}+\frac{R^{2} k}{n^{2 s}} .
$$

Furthermore, assuming that (8) holds,

$$
R^{2}\left(\frac{k}{n}\right)^{2 s} D_{k}^{*-2 s}+\frac{R^{2} k}{n^{2 s}}+\frac{\sqrt{D_{k}^{*}}}{n} \sigma^{2} \leq(7+\sqrt{2}) R^{\frac{2}{1+4 s}}\left(\frac{k}{n}\right)^{\frac{2 s}{1+4 s}}\left(\frac{\sigma^{2}}{n}\right)^{\frac{4 s}{4 s+1}},
$$


which ends the proof of Proposition 1.

Proof of Lemma 2: We have that

$$
\begin{aligned}
d_{n}^{2}\left(\bar{f}, S_{D}\right) & \leq \frac{1}{n} \sum_{j=1}^{n}\left(f(j / n)-\sum_{l=1}^{D} \beta_{l}(f) \varphi_{k / n, l}(j / n)\right)^{2} \\
& \leq \frac{1}{n}([n / k]+1) \sum_{j=1}^{k}\left(\sum_{l \geq D+1} \beta_{l}(f) \varphi_{k / n, l}(j / n)\right)^{2}
\end{aligned}
$$

Hence,

$$
\begin{aligned}
d_{n}^{2}\left(\bar{f}, S_{D}\right) \leq & \frac{2}{n}([n / k]+1) \sum_{j=1}^{k}\left(\sum_{l=D+1}^{k \vee(D+1)} \beta_{l}(f) \varphi_{k / n, l}(j / n)\right)^{2} \\
& +\frac{2}{n}([n / k]+1) \sum_{j=1}^{k}\left(\sum_{l \geq(k+1) \vee(D+2)} \beta_{l}(f) \varphi_{k / n, l}(j / n)\right)^{2} .
\end{aligned}
$$

We can see by straightforward computations that for $l, l^{\prime} \in\{1, \ldots, k\}, l \neq l^{\prime}$ :

$$
\sum_{j=1}^{k} \varphi_{k / n, l}(j / n) \varphi_{k / n, l^{\prime}}(j / n)=0
$$

So, we get

$$
d_{n}^{2}\left(\bar{f}, S_{D}\right) \leq \frac{4}{n}([n / k]+1) k \sum_{l \geq D+1} \beta_{l}^{2}(f)+\frac{4}{n}([n / k]+1) k \sum_{l \neq l^{\prime} \geq(k+1) \vee(D+2)}\left|\beta_{l}(f)\right|\left|\beta_{l^{\prime}}(f)\right|
$$

Therefore,

$$
d_{n}^{2}\left(\bar{f}, S_{D}\right) \leq 8\left(\sum_{l \geq D+1} \beta_{l}^{2}(f)+\left(\sum_{l \geq k+1}\left|\beta_{l}(f)\right|\right)^{2}\right)
$$

On the one hand, since

$$
\sum_{l \geq D+1} \beta_{l}^{2}(f)\left(\frac{l}{2}-\frac{1}{2}\right)^{2 s} \leq(2 \pi)^{-2 s} R^{2}\left(\frac{k}{n}\right)^{2 s}
$$

then for $D \geq 1$,

$$
\sum_{l \geq D+1} \beta_{l}^{2}(f) \leq(2 \pi)^{-2 s} R^{2}\left(\frac{k}{n}\right)^{2 s}\left(\frac{D}{2}\right)^{-2 s}
$$


On the other hand, by the Cauchy-Schwarz inequality,

$$
\begin{aligned}
\left(\sum_{l \geq k+1}\left|\beta_{l}(f)\right|\right)^{2} & \leq\left(\sum_{l \geq k+1} \beta_{l}^{2}(f)[l / 2]^{2 s}\right)\left(\sum_{l \geq k+1} \frac{1}{[l / 2]^{2 s}}\right) \\
& \leq(2 \pi)^{-2 s} R^{2}\left(\frac{k}{n}\right)^{2 s}\left(\sum_{l \geq k+1}\left(\frac{l}{2}-\frac{1}{2}\right)^{-2 s}\right) \\
& \leq(2 \pi)^{-2 s} R^{2}\left(\frac{k}{n}\right)^{2 s}\left(\sum_{l \geq k+1}\left(\frac{l}{4}\right)^{-2 s}\right) .
\end{aligned}
$$

Since $s$ is supposed to be larger than 1,

$$
\left(\sum_{l \geq k+1}\left|\beta_{l}(f)\right|\right)^{2} \leq R^{2}\left(\frac{k}{n}\right)^{2 s} k^{1-2 s} \leq R^{2} k n^{-2 s},
$$

and finally,

$$
d_{n}^{2}\left(\bar{f}, S_{D}\right) \leq C\left(R^{2}\left(\frac{k}{n}\right)^{2 s} D^{-2 s}+\frac{R^{2} k}{n^{2 s}}\right) .
$$

\subsection{Proof of Proposition 2}

The proof of Proposition 2 is based on a result due to Baraud, Huet and Laurent [2] that we recall here. Let $\bar{f}$ and $d_{n}$ respectively denote $(f(1 / n), \ldots, f(1))^{T}$ and the distance associated with the norm $\|\cdot\|_{\mathbb{R}^{n}}$.

Theorem 2 (Baraud, Huet, Laurent). Given some levels $\alpha$ and $\delta$ in $] 0,1\left[\right.$, let $T_{\alpha}$ be the test statistic defined by (9). Assume that $n \geq 2$ and that the collection $\left\{S_{m}, m \in \mathcal{M}\right\}$ satisfies:

$$
\left(\mathcal{H}_{\mathcal{M}}\right): \forall m \in \mathcal{M},\left\{\begin{array}{l}
u_{\alpha} \geq \mathrm{e}^{\left(\frac{D_{m}-n}{10}\right)} \\
\delta \geq 2 \mathrm{e}^{\left(\frac{D_{m}-n}{21}\right)}
\end{array}\right.
$$

Then,

for all $f$ satisfying

$$
\mathbb{P}_{f}\left(T_{\alpha}>0\right) \geq 1-\delta
$$

$$
\|f\|_{n}^{2} \geq \inf _{m \in \mathcal{M}}\left\{\begin{array}{c}
{\left[1+10\left(\frac{D_{m}+\log \left(1 / u_{\alpha}\right)}{n-D_{m}}\right)\right] d_{n}^{2}\left(\bar{f}, S_{m}\right)} \\
+\frac{\sigma^{2}}{n}\left[5\left(1+\sqrt{\frac{D_{m}}{n-D_{m}}}\right) \sqrt{D_{m} \log \left(\frac{2}{u_{\alpha} \delta}\right)}+\frac{25}{2}\left(1+2 \frac{D_{m}}{n-D_{m}}\right) \log \left(\frac{2}{u_{\alpha} \delta}\right)\right]
\end{array}\right\} .
$$

Since

$$
\begin{aligned}
\mathbb{P}_{0}\left(\sup _{m \in \mathcal{M}}\left\{\frac{\left(n-D_{m}\right)\left\|\Pi_{m}(Y)\right\|_{\mathbb{R}^{n}}^{2}}{D_{m}\left\|Y-\Pi_{m}(Y)\right\|_{\mathbb{R}^{n}}^{2}}-\bar{F}_{D_{m}, n-D_{m}}^{-1}\left(\frac{\alpha}{|\mathcal{M}|}\right)\right\}>0\right) \\
\leq \sum_{m \in \mathcal{M}} \mathbb{P}_{0}\left(\frac{\left(n-D_{m}\right)\left\|\Pi_{m}(Y)\right\|_{\mathbb{R}^{n}}^{2}}{D_{m}\left\|Y-\Pi_{m}(Y)\right\|_{\mathbb{R}^{n}}^{2}}>\bar{F}_{D_{m}, n-D_{m}}^{-1}\left(\frac{\alpha}{|\mathcal{M}|}\right)\right) \\
\leq \alpha,
\end{aligned}
$$


by the definition of $u_{\alpha}$, we have that $u_{\alpha} \geq \alpha /|\mathcal{M}|$. This implies that $u_{\alpha} \geq \alpha \log 2 / \log k$ and since $k \leq n$, under the assumptions of Proposition 2, $\left(\mathcal{H}_{\mathcal{M}}\right)$ is satisfied. Following Theorem 2, we then look for an upper bound for the quantity:

$$
\inf _{m \in \mathcal{M}}\left\{\begin{array}{c}
{\left[1+10\left(\frac{D_{m}+\log \left(1 / u_{\alpha}\right)}{n-D_{m}}\right)\right] d_{n}^{2}\left(\bar{f}, S_{m}\right)} \\
+\frac{\sigma^{2}}{n}\left[5\left(1+\sqrt{\frac{D_{m}}{n-D_{m}}}\right) \sqrt{D_{m} \log \left(\frac{2}{\delta u_{\alpha}}\right)}+\frac{25}{2}\left(1+2 \frac{D_{m}}{n-D_{m}}\right) \log \left(\frac{2}{u_{\alpha} \delta}\right)\right]
\end{array}\right\} .
$$

From the inequalities $u_{\alpha} \geq \alpha \log 2 / \log k$ and $D_{m} \leq m \leq n / 2$ for all $m \in \mathcal{M}$, together with $\left(\mathcal{H}_{\mathcal{M}}\right)$, we derive that we only need to find an upper bound for

$$
\inf _{D \in \mathcal{M}}\left\{c_{1} d_{n}^{2}\left(\bar{f}, S_{D}\right)+c_{2}(\alpha, \delta) \frac{\sqrt{D}}{n} \sqrt{\log \log k} \sigma^{2}\right\}+c_{3}(\alpha, \delta) \log \log k \frac{\sigma^{2}}{n} .
$$

Assuming that $f \in \mathcal{S}_{k}(s, R)$, Lemma 2 gives:

$$
d_{n}^{2}\left(\bar{f}, S_{D}\right) \leq C\left(R^{2}\left(\frac{k}{n}\right)^{2 s} D^{-2 s}+\frac{R^{2} k}{n^{2 s}}\right),
$$

and we obtain

$$
\begin{aligned}
\inf _{D \in \mathcal{M}}\left\{c_{1} d_{n}^{2}\left(\bar{f}, S_{D}\right)+\right. & \left.c_{2}(\alpha, \delta) \frac{\sqrt{D}}{n} \sqrt{\log \log k} \sigma^{2}\right\}+c_{3}(\alpha, \delta) \log \log k \frac{\sigma^{2}}{n} \\
& \leq \inf _{D \in \mathcal{M}}\left\{C_{1} R^{2}\left(\frac{k}{n}\right)^{2 s} D^{-2 s}+c_{2}(\alpha, \delta) \frac{\sqrt{D}}{n} \sqrt{\log \log k} \sigma^{2}\right\}+c_{3}(\alpha, \delta) \log \log k \frac{\sigma^{2}}{n}+C_{1} \frac{R^{2} k}{n^{2 s}}
\end{aligned}
$$

We can now compute the expected upper bound by noticing that

$$
R^{2}\left(\frac{k}{n}\right)^{2 s} D^{-2 s} \leq \frac{\sqrt{D}}{n} \sqrt{\log \log k} \sigma^{2} \text { and } D \in \mathcal{M} \quad \text { if and only if } \quad D \geq D^{*} \text { and } D \in \mathcal{M},
$$

where $D^{*}$ is the smallest dyadic integer at least equal to

$$
R^{\frac{4}{4 s+1}}\left(\frac{k}{n}\right)^{\frac{4 s}{4 s+1}} n^{\frac{2}{4 s+1}}(\sqrt{\log \log k})^{-\frac{2}{4 s+1}} \sigma^{-\frac{4}{4 s+1}}
$$

that is

$$
D^{*}=\inf \left\{2^{J}, J \in \mathbb{N}, 2^{J} \geq\left(R^{2}(k / n)^{2 s} n \sqrt{\log \log k}^{-1} \sigma^{-2}\right)^{2 /(4 s+1)}\right\} .
$$

If $D^{*} \in\left\{2, \ldots, 2^{\left[\log _{2}(k / 2)\right]}\right\}$, then

$$
\begin{aligned}
\inf _{D \in \mathcal{M}}\left\{C_{1} R^{2}\left(\frac{k}{n}\right)^{2 s} D^{-2 s}+c_{2}(\alpha, \delta)\right. & \left.\frac{\sqrt{D}}{n} \sqrt{\log \log k} \sigma^{2}\right\} \\
& \leq C_{1} R^{2}\left(\frac{k}{n}\right)^{2 s} D^{*-2 s}+c_{2}(\alpha, \delta) \frac{\sqrt{D^{*}}}{n} \sqrt{\log \log k} \sigma^{2} \\
& \leq\left(C_{1}+c_{2}(\alpha, \delta)\right) \frac{\sqrt{D^{*}}}{n} \sqrt{\log \log k} \sigma^{2} .
\end{aligned}
$$


Since $D^{*} \leq 2\left(R^{2}(k / n)^{2 s} n \sqrt{\log \log k}^{-1} \sigma^{-2}\right)^{\frac{2}{4 s+1}}$, this leads to

$$
\inf _{D \in \mathcal{M}}\left\{C_{1} R^{2}\left(\frac{k}{n}\right)^{2 s} D^{-2 s}+c_{2}(\alpha, \delta) \frac{\sqrt{D}}{n} \sqrt{\log \log k} \sigma^{2}\right\} \leq c(\alpha, \delta) R^{\frac{2}{4 s+1}}\left(\frac{k}{n}\right)^{\frac{2 s}{4 s+1}}\left(\frac{\sqrt{\log \log k} \sigma^{2}}{n}\right)^{\frac{4 s}{4 s+1}} .
$$

If $D^{*}=1$, then for all $D \in \mathcal{M}$,

$$
R^{2}\left(\frac{k}{n}\right)^{2 s} D^{-2 s} \leq \frac{\sqrt{D}}{n} \sqrt{\log \log k} \sigma^{2}
$$

and

$$
\inf _{D \in \mathcal{M}}\left\{C_{1} R^{2}\left(\frac{k}{n}\right)^{2 s} D^{-2 s}+c_{2}(\alpha, \delta) \frac{\sqrt{D}}{n} \sqrt{\log \log k} \sigma^{2}\right\} \leq c^{\prime}(\alpha, \delta) \sqrt{\log \log k} \frac{\sigma^{2}}{n} .
$$

If $D^{*}>2^{\left[\log _{2}(k / 2)\right]}$, then for all $D \in \mathcal{M}$,

$$
\frac{\sqrt{D}}{n} \sqrt{\log \log k} \sigma^{2}<R^{2}\left(\frac{k}{n}\right)^{2 s} D^{-2 s},
$$

and

$$
\begin{aligned}
\inf _{D \in \mathcal{M}}\left\{C_{1} R^{2}\left(\frac{k}{n}\right)^{2 s} D^{-2 s}+c_{2}(\alpha, \delta) \frac{\sqrt{D}}{n} \sqrt{\log \log k} \sigma^{2}\right\} & \leq c(\alpha, \delta) R^{2}\left(\frac{k}{n}\right)^{2 s}\left(2^{\left[\log _{2}(k / 2)\right]}\right)^{-2 s} \\
& \leq 2^{4 s} c(\alpha, \delta) \frac{R^{2}}{n^{2 s}}
\end{aligned}
$$

So, in all cases

$$
\begin{array}{r}
\inf _{D \in \mathcal{M}}\left\{C_{1} R^{2}\left(\frac{k}{n}\right)^{2 s}\right. \\
\left.D^{-2 s}+c_{2}(\alpha, \delta) \frac{\sqrt{D}}{n} \sqrt{\log \log k} \sigma^{2}\right\}+c_{3}(\alpha, \delta) \log \log k \frac{\sigma^{2}}{n}+C_{1} \frac{R^{2} k}{n^{2 s}} \\
\leq c(\alpha, \delta) R^{\frac{2}{4 s+1}}\left(\frac{k}{n}\right)^{\frac{2 s}{4 s+1}}\left(\frac{\sqrt{\log \log k} \sigma^{2}}{n}\right)^{\frac{4 s}{4 s+1}}+c^{\prime}(\alpha, \delta) \frac{\sqrt{\log \log k} \sigma^{2}}{n} \\
+c(s, \alpha, \delta) \frac{R^{2}}{n^{2 s}}+c_{3}(\alpha, \delta) \frac{\log \log k \sigma^{2}}{n}+C_{1} \frac{R^{2} k}{n^{2 s}}
\end{array}
$$

which gives the result.

\subsection{Proof of Proposition 3}

As in the proof of Proposition 2, since $u_{\alpha} \geq \alpha /|\mathcal{M}|$, under the assumptions of Proposition $3,\left(\mathcal{H}_{\mathcal{M}}\right)$ is satisfied and we look for an upper bound for

$$
\inf _{(k, D) \in \mathcal{M}}\left\{c_{1} d_{n}^{2}\left(\bar{f}, S_{(k, D)}\right)+c_{2}(\alpha, \delta) \sqrt{D \log n} \frac{\sigma^{2}}{n}\right\}+c_{3}(\alpha, \delta) \log n \frac{\sigma^{2}}{n}
$$

when $f \in \mathcal{S}\left(s, R, \tau_{1}\right)$, for $s \in \mathbb{N}^{*}, R>0, \tau_{1} \in[2 / n, 1]$. 
Assume that $f \in \mathcal{S}\left(s, R, \tau_{1}\right)$. Let $k(f)=[\tau(f) n]+1$ if $\tau(f) n \notin \mathbb{N}^{*}, \tau(f) n$ if $\tau(f) n \in \mathbb{N}^{*}$. Then $k(f) \in$ $\{2, \ldots, n\}$ and

$$
\begin{aligned}
\inf _{(k, D) \in \mathcal{M}}\left\{c_{1} d_{n}^{2}\left(\bar{f}, S_{(k, D)}\right)+c_{2}(\alpha, \delta) \sqrt{D \log n} \frac{\sigma^{2}}{n}\right\} \leq & \\
& \inf _{D \in\left\{1, \ldots, 2^{\left[\log _{2}(k(f) / 2)\right]}\right\}}\left\{c_{1} d_{n}^{2}\left(\bar{f}, S_{(k(f), D)}\right)+c_{2}(\alpha, \delta) \sqrt{D \log n} \frac{\sigma^{2}}{n}\right\} .
\end{aligned}
$$

Denote by $\beta_{l}(f)$ the $l$-th Fourier coefficient of $f$. Recall that if $f \in \mathcal{S}\left(s, R, \tau_{1}\right)$, for all $x \in[0,1], f(x)=$ $\beta_{1}(f)+\sum_{l \geq 2} \beta_{l}(f) \varphi_{\tau(f), l}(x)$ with

$$
(2 \pi)^{2 s} \sum_{l \geq 2}[l / 2]^{2 s} \beta_{l}^{2}(f) \leq R^{2} \tau(f)^{2 s}
$$

and $\sum_{l \geq 1}\left|\beta_{l}(f)\right|<+\infty$. By introducing $\tilde{f}$ such that $\tilde{f}(x)=\beta_{1}(f)+\sum_{l \geq 2} \beta_{l}(f) \varphi_{k(f) / n, l}(x)$, we have:

$$
d_{n}^{2}\left(\bar{f}, S_{(k(f), D)}\right) \leq 2\left(d_{n}^{2}(\bar{f}, \overline{\tilde{f}})+d_{n}^{2}\left(\overline{\tilde{f}}, S_{(k(f), D)}\right)\right)
$$

where $\overline{\tilde{f}}=(\tilde{f}(1 / n), \ldots, \tilde{f}(1))^{T}$.

On the one hand, since $\tau(f) \leq k(f) / n,(23)$ implies that $\tilde{f}$ satisfies the assumptions of Lemma 2 with $k=k(f)$, so:

$$
\begin{aligned}
d_{n}^{2}\left(\overline{\tilde{f}}, S_{(k(f), D)}\right) & \leq C\left(R^{2}(k(f) / n)^{2 s} D^{-2 s}+R^{2} k(f) n^{-2 s}\right) \\
& \leq c(s)\left(R^{2} \tau(f)^{2 s} D^{-2 s}+R^{2} \tau(f) n^{1-2 s}\right) .
\end{aligned}
$$

On the other hand, using the Cauchy-Schwarz inequality,

$$
\begin{aligned}
d_{n}^{2}(\bar{f}, \overline{\tilde{f}}) & =\frac{1}{n} \sum_{j=1}^{n}\left(\sum_{l \geq 2} \beta_{l}(f)\left[\varphi_{\tau(f), l}(j / n)-\varphi_{k(f) / n, l}(j / n)\right]\right)^{2} \\
& \leq \frac{8}{n} \sum_{j=1}^{n}\left(\sum_{l \geq 2}[l / 2]^{2 s} \beta_{l}^{2}(f)\right)\left(\sum_{l \geq 2}[l / 2]^{-2 s}\left|\sin \left(\frac{\pi j[l / 2]}{n \tau(f)}-\frac{\pi j[l / 2]}{k(f)}\right)\right|^{2}\right) .
\end{aligned}
$$

Let $\gamma \in\left[0,1\left[\right.\right.$ that will be chosen later. For all $x \in \mathbb{R}, \sin ^{2}(x) \leq|x|^{\gamma}$, which implies that:

$$
\begin{aligned}
d_{n}^{2}(\bar{f}, \overline{\tilde{f}}) & \leq \frac{c(s)}{n} R^{2} \tau(f)^{2 s} \sum_{j=1}^{n}\left(\sum_{l \geq 2} j^{\gamma}[l / 2]^{\gamma-2 s} \frac{1}{(\tau(f) n)^{2 \gamma}}\right) \\
& \leq c(s) \frac{R^{2} \tau(f)^{2 s-2 \gamma}}{n^{2 \gamma+1}}\left(\sum_{j=1}^{n} j^{\gamma}\right)\left(\sum_{l \geq 2}[l / 2]^{\gamma-2 s}\right) \\
& \leq \frac{c^{\prime}(s)}{2 s-\gamma-1} \frac{R^{2} \tau(f)^{2 s-2 \gamma}}{n^{\gamma}} .
\end{aligned}
$$


Then, we have:

$$
\begin{aligned}
& \inf _{(k, D) \in \mathcal{M}}\left\{c_{1} d_{n}^{2}\left(\bar{f}, S_{(k, D)}\right)+c_{2}(\alpha, \delta) \sqrt{D \log n} \frac{\sigma^{2}}{n}\right\}+c_{3}(\alpha, \delta) \log n \frac{\sigma^{2}}{n} \\
& \inf _{D \in\left\{1, \ldots, 2^{\left[\log _{2}(k(f) / 2)\right]}\right\}}\left\{c_{1}(s) R^{2} D^{-2 s} \tau(f)^{2 s}+c_{2}(\alpha, \delta) \sqrt{D \log n} \frac{\sigma^{2}}{n}\right\} \\
& +c_{3}(\alpha, \delta) \log n \frac{\sigma^{2}}{n}+\frac{c_{4}(s)}{2 s-\gamma-1} \frac{R^{2} \tau(f)^{2 s-2 \gamma}}{n^{\gamma}}+c_{1}(s) \frac{R^{2} \tau(f)}{n^{2 s-1}} .
\end{aligned}
$$

Note again that $R^{2} D^{-2 s} \tau(f)^{2 s} \leq \sqrt{D \log n} \sigma^{2} / n$ and $D$ is dyadic if and only if $D \geq D^{*}(f)$, where $D^{*}(f)$ is the smallest dyadic integer at least equal to $\left(R^{2} \tau(f)^{2 s} n \sigma^{-2}(\log n)^{-1 / 2}\right)^{2 /(4 s+1)}$, that is

$$
D^{*}(f)=\inf \left\{2^{J}, J \in \mathbb{N}, 2^{J} \geq\left(R^{2} \tau(f)^{2 s} n \sqrt{\log n}^{-1} \sigma^{-2}\right)^{2 /(4 s+1)}\right\}
$$

If $D^{*}(f) \in\left\{2, \ldots, 2^{\left[\log _{2}(k(f) / 2)\right]}\right\}$, then

$$
D^{*}(f) \leq 2\left(\frac{R^{2} \tau(f)^{2 s} n}{\sigma^{2} \sqrt{\log n}}\right)^{\frac{2}{4 s+1}}
$$

and

$$
\begin{aligned}
\inf _{D \in\left\{1, \ldots, 2^{\left[\log _{2}(k(f) / 2)\right]}\right\}}\left\{c_{1}(s) R^{2} D^{-2 s} \tau(f)^{2 s}+c_{2}(\alpha, \delta) \sqrt{D \log n} \frac{\sigma^{2}}{n}\right\} & \\
\leq c_{1}(s) R^{2} D^{*}(f)^{-2 s} \tau(f)^{2 s}+c_{2}(\alpha, \delta) \sqrt{D^{*}(f) \log n} \frac{\sigma^{2}}{n} & \leq c(s, \alpha, \delta)\left(R^{2} \tau(f)^{2 s}\right)^{\frac{1}{4 s+1}}\left(\sqrt{\log n} \frac{\sigma^{2}}{n}\right)^{\frac{4 s}{4 s+1}} .
\end{aligned}
$$

If $D^{*}(f)=1$, then for all $D \in\left\{1, \ldots, 2^{\left[\log _{2}(k(f) / 2)\right]}\right\}, R^{2} D^{-2 s} \tau(f)^{2 s} \leq \sqrt{D \log n} \sigma^{2} / n$ and

$$
\begin{aligned}
\inf _{D \in\left\{1, \ldots, 2^{\left[\log _{2}(k(f) / 2)\right]}\right\}}\left\{c_{1}(s) R^{2} D^{-2 s} \tau(f)^{2 s}\right. & \left.+c_{2}(\alpha, \delta) \sqrt{D \log n} \frac{\sigma^{2}}{n}\right\} \\
& \leq c^{\prime}(s, \alpha, \delta) \inf _{D \in\left\{1, \ldots, 2^{\left[\log _{2}(k(f) / 2)\right]}\right\}}\left\{\sqrt{D \log n} \frac{\sigma^{2}}{n}\right\} \leq c^{\prime}(s, \alpha, \delta) \sqrt{\log n} \frac{\sigma^{2}}{n} .
\end{aligned}
$$

At last, if $D^{*}(f)>2^{\left[\log _{2}(k(f) / 2)\right]}$, for all $D \in\left\{1, \ldots, 2^{\left[\log _{2}(k(f) / 2)\right]}\right\}, R^{2} D^{-2 s} \tau(f)^{2 s}>\sqrt{D \log n} \sigma^{2} / n$, which leads to:

$$
\inf _{D \in\left\{1, \ldots, 2^{\left[\log _{2}(k(f) / 2)\right]}\right\}}\left\{c_{1}(s) R^{2} D^{-2 s} \tau(f)^{2 s}+c_{2}(\alpha, \delta) \sqrt{D \log n} \frac{\sigma^{2}}{n}\right\} \leq c^{\prime \prime}(s, \alpha, \delta) R^{2} n^{-2 s}
$$


Finally,

$$
\begin{gathered}
\inf _{(k, D) \in \mathcal{M}}\left\{c_{1} d_{n}^{2}\left(\bar{f}, S_{(k, D)}\right)+c_{2}(\alpha, \delta) \sqrt{D \log n} \frac{\sigma^{2}}{n}\right\}+c_{3}(\alpha, \delta) \log n \frac{\sigma^{2}}{n} \\
\leq C(s, \alpha, \delta)\left\{\left(R^{2} \tau_{1}^{2 s}\right)^{\frac{1}{4 s+1}}\left(\sqrt{\log n} \frac{\sigma^{2}}{n}\right)^{\frac{4 s}{4 s+1}}+\log n \frac{\sigma^{2}}{n}+\frac{R^{2} \tau_{1}}{n^{2 s-1}}\right. \\
\left.+\frac{R^{2}}{n^{2 s}}+\frac{1}{2 s-\gamma-1} \frac{R^{2} \tau_{1}^{2 s}}{\left(n \tau_{1}^{2}\right)^{\gamma}}\right\}
\end{gathered}
$$

Since $n^{-1} \leq \tau_{1} \leq 1$, we can see that for all $\gamma \in\left[0,1\left[\right.\right.$, for all $s \in \mathbb{N}^{*}$,

$$
\frac{R^{2}}{n^{2 s}} \leq \frac{R^{2} \tau_{1}}{n^{2 s-1}} \leq \frac{R^{2} \tau_{1}^{2 s}}{\left(n \tau_{1}^{2}\right)^{\gamma}}
$$

To prove the final result, we need to distinguish between two cases in order to optimize the choice of $\gamma$. If $n \tau_{1}^{2} \leq 2$, by taking $\gamma=0$, we obtain:

$$
\frac{1}{2 s-\gamma-1} \frac{R^{2} \tau_{1}^{2 s}}{\left(n \tau_{1}^{2}\right)^{\gamma}}=\frac{1}{2 s-1} R^{2} \tau_{1}^{2 s}=\frac{1}{2 s-1}\left(2 \frac{R^{2} \tau_{1}^{2 s}}{n \tau_{1}^{2}} \wedge R^{2} \tau_{1}^{2 s}\right)
$$

If $n \tau_{1}^{2}>2$, by taking $\gamma_{n}=1-\log 2 / \log \left(n \tau_{1}^{2}\right)$, we get:

$$
\frac{1}{2 s-1-\gamma_{n}} \frac{R^{2} \tau_{1}^{2 s}}{\left(n \tau_{1}^{2}\right)^{\gamma_{n}}} \leq \frac{2}{2 s-1-\gamma_{n}} \frac{R^{2} \tau_{1}^{2 s}}{n \tau_{1}^{2}} \leq \frac{2 \log n}{\log 2} \frac{R^{2} \tau_{1}^{2 s}}{n \tau_{1}^{2}} \leq \frac{2 \log n}{\log 2}\left(2 \frac{R^{2} \tau_{1}^{2 s}}{n \tau_{1}^{2}} \wedge R^{2} \tau_{1}^{2 s}\right)
$$

which ends the proof.

\section{Appendix: The Gaussian White nOISE MODEL}

In this section, we consider the following ideal Gaussian white noise model instead of our current regression framework:

$$
\mathrm{d} Y(t)=f(t) \mathrm{d} t+\frac{1}{\sqrt{n}} \mathrm{~d} W(t), t \in[0,1]
$$

Let $\tau$ such that $1 / \tau$ is an integer. Assume that $f$ belongs to $\mathbb{L}_{2}([0,1])$ and that $f$ is periodic with period $\tau$. By projecting the above model onto the Fourier basis $\left\{\varphi_{\tau, l}, l \geq 1\right\}$ defined by (5) on [0,1], we obtain the Gaussian sequence model:

$$
Y_{l}=\beta_{l}(f)+\frac{\varepsilon_{l}}{\sqrt{n}}, l \in \mathbb{N}^{*},
$$

where

$$
Y_{l}=\int_{0}^{1} \varphi_{\tau, l}(t) \mathrm{d} Y(t), \beta_{l}(f)=\int_{0}^{1} \varphi_{\tau, l}(t) f(t) \mathrm{d} t, \varepsilon_{l}=\int_{0}^{1} \varphi_{\tau, l}(t) \mathrm{d} W(t) .
$$

Testing the null hypothesis " $f=0$ " against " $f \neq 0$ " in the model $(24)$ is equivalent to testing " $\beta_{l}(f)=0, \forall l \geq 1$ " against " $\exists l \geq 1, \beta_{l}(f) \neq 0$ " in the model $(25)$. 
Let $\mathcal{E}(\tau, R)$ be the ellipsoid defined by

$$
\mathcal{E}(\tau, R)=\left\{\left(\beta_{l}(f), l \geq 1\right), \sum_{l \geq 2} \frac{\beta_{l}^{2}(f)}{\left([l / 2]^{-s}\right)^{2}} \leq(2 \pi)^{-2 s} R^{2} \tau^{2 s}\right\} .
$$

Considering the problem of testing " $\beta_{l}(f)=0, \forall l \geq 1$ " in the model (25), Baraud has comprehensively studied the minimax separation rate $\bar{\rho}$ over such an ellipsoid with respect to the classical norm of $l^{2}(\mathbb{N})$. From his results, we can deduce the following bounds:

$$
\bar{\rho}^{2}(\mathcal{E}(\tau, R), \alpha, \delta) \geq \sup _{D \geq 1}\left\{\left(\frac{\sqrt{2 \mathcal{L}(\alpha, \delta) D}}{n}\right) \wedge\left((2 \pi)^{-2 s} R^{2} \tau^{2 s}[D / 2]^{-2 s}\right)\right\}
$$

where $\mathcal{L}(\alpha, \delta)=\log \left(1+4(1-\alpha-\delta)^{2}\right)$, and if $R>\tau^{-s} n^{-1 / 2}$,

$$
\bar{\rho}^{2}(\mathcal{E}(\tau, R), \alpha, \delta) \leq c(s, \alpha, \delta) \sup _{D \geq 1}\left\{\frac{\sqrt{D}}{n} \wedge R^{2} \tau^{2 s} D^{-2 s}\right\} \leq 2 c(s, \alpha, \delta) R^{\frac{2}{4 s+1}} \tau^{\frac{2 s}{4 s+1}} n^{-\frac{4 s}{4 s+1}} .
$$

Since $\sum_{l \geq 1} \beta_{l}^{2}(f)=\tau^{-1} \int_{0}^{\tau} f^{2}(t) \mathrm{d} t=\|f\|_{2, \tau}^{2}$, we thus find lower bounds similar to the ones obtained in our Gaussian periodic fixed design regression framework over the classes $\mathcal{S}_{k}^{\prime}(s, R)=\{f, f$ is periodic with period $k / n$ and $f(x)=\sum_{l \geq 1} \beta_{l}(f) \varphi_{k / n, l}(x)$ with $\left.(2 \pi)^{2 s} \sum_{l \geq 2}[l / 2]^{2 s} \beta_{l}^{2}(f) \leq R^{2}(k / n)^{2 s}\right\}$ (see Th. 1 ), except that the supremum over $D \geq 1$ is replaced by a supremum over $D \in\{1, \ldots,[\kappa k]\}$. As for the upper bounds, we can see via Proposition 1, that if $R>(k / n)^{-s} n^{-1 / 2}$,

$$
\tilde{\rho}^{2}\left(\mathcal{S}_{k}^{\prime}(s, R), \alpha, \delta, \sigma\right) \leq 2 c(\alpha, \delta)\left(R^{\frac{2}{1+4 s}}\left(\frac{k}{n}\right)^{\frac{2 s}{4 s+1}}\left(\frac{\sigma^{2}}{n}\right)^{\frac{4 s}{4 s+1}}+\frac{R^{2} k}{n^{2 s}}\right) .
$$

The losses that one can observe are essentially due to the fact that the Fourier family $\left\{\varphi_{k / n, l}, l \geq 1\right\}$ is not orthogonal with respect to the norm $\|\cdot\|_{n}$ that we consider.

\section{REFERENCES}

[1] Y. Baraud, Non-asymptotic minimax rates of testing in signal detection. Bernoulli 8 (2002) 577-606.

[2] Y. Baraud, S. Huet, and B. Laurent, Adaptive tests of linear hypotheses by model selection. Ann. Statist. 31 (2003) 225-251.

[3] L. Birgé, An alternative point of view on Lepski's method, in State of the Art in Probability and Statistics (Leiden, 1999), 113-133, IMS Lecture Notes Monogr. Ser. 36 (2000).

[4] P.J. Brockwell and R.A. Davis, Time series: theory and methods. Springer Series in Statistics. Springer-Verlag, New York, second edition (1991).

[5] R. Eubank and J. Hart, Testing goodness-of-fit in regression via order selection criteria. Ann. Stat. 20 (1992) 1412-1425.

[6] J. Fan and Q. Yao, Nonlinear Time series. Springer series in Statistics. Springer-Verlag, New York, Nonparametric and parametric methods (2003).

[7] G. Gayraud and C. Pouet, Minimax testing composite null hypotheses in the discrete regression scheme. Math. Methods Stat. 10 (2001) 375-394.

[8] P. Gregory and T. Loredo, A new method for the detection of a periodic signal of unknown shape and period. The Astrophysical J. 398 (1992) 146-168.

[9] W. Härdle and A. Kneip, Testing a regression model when we have smooth alternatives in mind. Scand. J. Stat. 26 (1999) $221-238$.

[10] J. Horowitz and V. Spokoiny, An adaptive, rate-optimal test of a parametric mean-regression model against a nonparametric alternative. Econometrica 69 (2001) 599-631.

[11] Y. Ingster, Minimax nonparametric detection of signals in white Gaussian noise. Probl. Inf. Transm. 18 (1982) 130-140.

[12] Y. Ingster, Asymptotically minimax testing for nonparametric alternatives I-II-III. Math. Methods Statist. 2 (1993) 85-114, 171-189, 249-268. 
[13] B. Laurent and P. Massart, Adaptive estimation of a quadratic functional by model selection. Ann. Statist. 28 (2000) $1302-1338$.

[14] M. Lavielle and C. Lévy-Leduc, Semiparametric estimation of the frequency of unknown periodic functions and its application to laser vibrometry signals. IEEE Trans. Signal Proces. 53 (2005) 2306-2314.

[15] O. Lepski and V. Spokoiny, Minimax nonparametric hypothesis testing: The case of an inhomogeneous alternative. Bernoulli 5 (1999) 333-358.

[16] O. Lepski and A. Tsybakov, Asymptotically exact nonparametric hypothesis testing in sup-norm and at a fixed point. Probab. Theory Relat. Fields 117 (2000) 17-48.

[17] M. Prenat, Vibration modes and laser vibrometry performance in noise, in Proceedings of the Physics in Signal and Image Processing conference (PSIP'01), 23-24 janvier 2001, Marseille, France (2001).

[18] B.G. Quinn and E.J. Hannan, The estimation and tracking of frequency. Cambridge Series in Statistical and Probabilistic Mathematics. Cambridge University Press, Cambridge (2001).

[19] V. Spokoiny, Adaptive hypothesis testing using wavelets. Ann. Stat. 24 (1996) 2477-2498.

[20] V. Spokoiny, Adaptive and spatially adaptive testing of a nonparametric hypothesis. Math. Methods Stat. 7 (1998) $245-273$. 